\title{
Determinants of saxagliptin use among patients with type 2 diabetes mellitus treated with oral anti-diabetic drugs
}

M Elle Saine ${ }^{1,2}$, Dena M Carbonari ${ }^{1,2}$, Craig W Newcomb ${ }^{1}$, Melissa S Nezamzadeh ${ }^{1,2}$, Kevin Haynes ${ }^{1,2,3}$, Jason A Roy ${ }^{1,2}$, Serena Cardillo ${ }^{4}$, Sean Hennessy ${ }^{1,2}$, Crystal N Holick $^{3}$, Daina B Esposito ${ }^{3}$, Arlene M Gallagher ${ }^{5}$, Harshvinder Bhullar ${ }^{6}$, Brian L Strom ${ }^{1,2,7}$ and Vincent Lo Re III ${ }^{1,2,4^{*}}$

\begin{abstract}
Background: The patterns and determinants of saxagliptin use among patients with type 2 diabetes mellitus (T2DM) are unknown in real-world settings. We compared the characteristics of T2DM patients who were new initiators of saxagliptin to those who were new initiators of non-dipeptidyl peptidase-4 (DPP-4) inhibitor oral anti-diabetic drugs (OADs) and identified factors associated with saxagliptin use.

Methods: We conducted a cross-sectional study within the Clinical Practice Research Datalink (CPRD), The Health Improvement Network (THIN), US Medicare, and the HealthCore Integrated Research Database (HIRD ${ }^{S M}$ ) across the first 36 months of saxagliptin availability (29 months for US Medicare). Patients were included if they were: 1) $\geq 18$ years old, 2) newly prescribed saxagliptin or a non-DPP-4 inhibitor OAD, and 3) enrolled in their respective database for 180 days. For each saxagliptin initiator, we randomly selected up to ten non-DPP-4 inhibitor OAD initiators matched on age, sex, and geographic region. Conditional logistic regression was used to identify determinants of saxagliptin use.

Results: We identified 64,079 saxagliptin initiators (CPRD: 1,962; THIN: 2,084; US Medicare: 51,976; HIRD ${ }^{\text {SM: }}$ 8,057) and

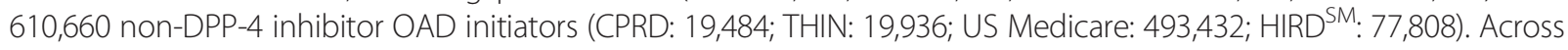
all four data sources, prior OAD use, hypertension, and hyperlipidemia were associated with saxagliptin use. Saxagliptin initiation was also associated with hemoglobin A1c results $>8 \%$ within the UK data sources, and a greater number of hemoglobin A1c measurements in the US data sources.
\end{abstract}

Conclusions: In these UK and US data sources, initiation of saxagliptin was associated with prior poor glycemic control, prior OAD use, and diagnoses of hypertension and hyperlipidemia.

Trial registration: ClinicalTrials.gov identifiers NCT01086280, NCT01086293, NCT01086319, NCT01086306, and NCT01377935

Keywords: Diabetes mellitus, Saxagliptin, Dipeptidyl peptidase IV inhibitor, Pharmacoepidemiology

\footnotetext{
* Correspondence: vincent|@mail.med.upenn.edu

${ }^{1}$ Department of Biostatistics and Epidemiology, Center for Clinical

Epidemiology and Biostatistics, Perelman School of Medicine at the

University of Pennsylvania, 423 Guardian Drive, Philadelphia, PA, USA

${ }^{2}$ Department of Biostatistics and Epidemiology, Center for

Pharmacoepidemiology Research and Training, Perelman School of Medicine

at the University of Pennsylvania, Philadelphia, PA, USA

Full list of author information is available at the end of the article
} 


\section{Background}

Type 2 diabetes mellitus (T2DM) is a global public health problem, affecting 347 million people worldwide [1,2]. Current estimates suggest that more than 29.1 million adults and children in the United States (US) and 3.2 million adults and children in the United Kingdom (UK) have T2DM, representing more than $9.3 \%$ and $6 \%$ of these populations, respectively [3-5]. Oral anti-diabetic drugs (OADs), along with diet and exercise, can help to control T2DM-associated hyperglycemia in adults [6].

Saxagliptin, a relatively new dipeptidyl peptidase-4 (DPP-4) inhibitor [7], was approved by the US Food and Drug Administration (FDA) in July 2009 and the European Medicines Agency in October 2009 to be used with diet and exercise to control hyperglycemia in adults with T2DM. In clinical trials, saxagliptin was shown to be efficacious in lowering fasting plasma glucose, 2-hour postprandial glucose, and hemoglobin A1c when used as monotherapy [8], in combination with metformin in treatment-naive patients [9], or as add-on therapy to metformin [10], sulfonylureas [11], thiazolidinediones [12], or insulin [13]. Because of its recent market introduction, prescribing patterns associated with saxagliptin's use in real-world settings remain unknown. Determining how OADs are prescribed in clinical practice can provide valuable information on healthcare decision-making [14,15]. Further, since the effectiveness and safety of saxagliptin and other OAD therapies may be affected by demographic characteristics, medical comorbidities, and additional medications prescribed to T2DM patients, identifying the factors associated with the use of particular OADs in real-world settings can provide important information needed for the future conduct of studies evaluating the comparative effectiveness and safety of anti-diabetic drugs. In particular, such variables can be incorporated within propensity scores to help to minimize confounding by indication $[16,17]$.

The objectives of this study were to: 1) compare the characteristics of patients with T2DM who newly initiate saxagliptin to those who newly initiate OADs in classes other than DPP-4 inhibitors, and 2) identify determinants of saxagliptin use during the first years of its availability in the UK and US. We hypothesized that T2DM patients with poor glycemic control, a higher prevalence of microvascular and macrovascular complications, and prior OAD use would be more likely to initiate saxagliptin.

\section{Methods}

\section{Data sources}

Four data sources, two each in the UK and US, were used in this study. Within the UK, data from the Clinical Practice Research Datalink (CPRD; formerly General Practice Research Database) and The Health Improvement Network (THIN) were evaluated over the first 36 months of saxagliptin availability (5 October 2009 to 30 September 2012). Within the US, data from US Medicare were evaluated across all 50 states over the first 29 months of saxagliptin availability (1 August 2009 to 31 December 2011), due to the lag in availability of these data. The HealthCore Integrated Research Database $\left(\mathrm{HIRD}^{\mathrm{SM}}\right.$ ) data were examined over the first 36 months of saxagliptin availability (1 August 2009 to 31 July 2012). These four databases were selected because they include large numbers of T2DM patients across all age groups and utilize health records (UK) and claims data (US) from both private and public insurance plans, providing broadly representative study samples.

Details on the data available within each of these data sources for the purpose of evaluating OAD use have been previously described [18]. At the time of data collection, CPRD contains electronic medical records of over 15 million UK patients across 684 practices [19], and THIN contained primary medical records for over 11 million UK patients across over 550 practices [20,21]. CPRD and THIN collect demographic information, medical diagnoses and surgical procedures (recorded using Read codes), outpatient laboratory results, and general practitioner-issued prescriptions [22,23]. Since some UK practices contribute data to both CPRD and THIN [24], we excluded overlapping patients from THIN data to ensure that these patients were not counted twice. US Medicare is the largest national health insurance program administered by the US federal government, serving approximately 47.5 million people as of 2010 [25]. Medicare is available to US citizens aged 65 years or older and those under 65 years with certain disabilities. The HIRD ${ }^{\mathrm{SM}}$ is one of the largest longitudinal commercial health insurance databases in the US, serving 23.2 million members as of 2010 [26-28]. Both Medicare and the HIRD ${ }^{\mathrm{SM}}$ contain demographic information, inpatient and outpatient medical diagnoses (recorded using International Classification of Diseases, Ninth Revision, Clinical Modification diagnosis codes), surgical procedures (recorded with Current Procedural Terminology codes), and dispensed medications (recorded by National Drug Codes). Although codes for ordered laboratory tests can be identified within Medicare and the HIRD $^{\mathrm{SM}}$, the results of these tests are not recorded in Medicare and are only available in a subset of HIRD $^{\text {SM }}$ patients. To avoid the possibility of doublecounting patients concurrently enrolled in both of these US data sources, we only included HIRD ${ }^{\mathrm{SM}}$ data for persons aged 18-64 years and censored HIRD ${ }^{\mathrm{SM}}$ enrollees at age 65 years.

The study was approved by the University of Pennsylvania and Rutgers University Institutional Review Boards, the Quorum Review Institutional Review Board (HIRD ${ }^{\mathrm{SM}}$ ), and the Independent Scientific Advisory Committees for 
CPRD and THIN. A data use agreement was obtained from the Centers for Medicare and Medicaid Services (US Medicare).

\section{Study patients}

Patients were eligible for study inclusion if they were: 1 ) newly prescribed (in the UK) or dispensed (in the US) either saxagliptin, as a single agent or in combination with other OADs, or an OAD in a class other than DPP-4 inhibitors ("index drug"); 2) $\geq 18$ years old; and 3) enrolled in their respective data source for at least 180 days prior to initiation of their index drug. The rationale for selecting new initiators of other OADs as the comparator group was to study patients with diabetes who required initiation of new OAD therapy. Given that we wished to identify factors specifically associated with saxagliptin use, we did not include new initiators of other DPP-4 inhibitors within the comparator OAD group.

All eligible patients prescribed saxagliptin were included. Within each data source, a random sample (without replacement) of up to ten new initiators of non-DPP-4 inhibitor OADs was selected for each saxagliptin initiator. These patients were matched on age (within 5-year age groups), sex, and geographic region (i.e., country within UK data sources; census region within US data sources).

\section{Main study outcome}

The main study outcome was a new prescription (UK data source) or pharmacy claim (US data source) for either saxagliptin or a non-DPP-4 inhibitor OAD. The index date was defined as the date of first prescription of saxagliptin or comparator $\mathrm{OAD}$ in the respective data source.

\section{Determinants of saxagliptin use}

The following variables were evaluated as determinants of use of saxagliptin compared to other OADs: calendar year of initiation, medical comorbidities, surgical procedures, and medications of interest (listed in Table 1). In clinical practice, primary care physicians and endocrinologists likely select and prescribe oral anti-diabetic drugs based on consideration of at least many of these factors. We included a variety of medications and drug classes as potential determinants of saxagliptin because concerns for drug-drug interactions or exacerbation of medication toxicities might influence decisions to prescribe saxagliptin.

Comorbidities were identified based on the presence of diagnoses recorded in the 180 days prior to the index date within US Medicare and HIRD ${ }^{\mathrm{SM}}$, and at any time prior to the index date within CPRD and THIN. General practitioners in the UK do not have a financial incentive to record pre-existing diagnoses at each visit and only utilizing diagnoses recorded in the 180 days prior to the index date could lead to incomplete comorbidity ascertainment within the UK data sources. Within all data sources, pre-existing microvascular and macrovascular T2DM complications were determined based on diagnoses and surgical procedures recorded within 180 days prior to the index date and categorized according to the Diabetes Complications Severity Index [29].

Within the UK data sources, we collected the closest hemoglobin A1c result recorded in the 180 days prior to the index date. Smoking history and obesity, defined as body mass index (calculated as height in meters/[body weight in $\mathrm{kg}]^{2}$ ) $>30 \mathrm{~kg} / \mathrm{m}^{2}$, were also extracted from CPRD and THIN. Within the US data sources, we collected the number of claims for hemoglobin A1c tests recorded in the 180 days prior to the index date, since patients with unmanaged and/or severe T2DM typically have hemoglobin A1c measured more frequently [30].

Across all data sources, patients were considered exposed to a particular drug if a prescription or pharmacy claim for that drug was recorded within 180 days prior to the index date. Particularly, prior OAD use within the 180 days preceding the index date was determined. Patients whose prescriptions or claims for their existing OAD therapy continued for 90 days before and after the initiation of their index drug were considered to have "added on" saxagliptin or the comparator OAD to their current therapy. Patients whose prescriptions or claims for their existing OAD therapy were recorded 90 days before, but not after, the initiation of the index drug were considered to have "switched to" saxagliptin or a comparator OAD.

\section{Statistical analysis}

Baseline characteristics of new initiators of saxagliptin or other OADs were compared using standardized differences, of which a value exceeding 0.1 is generally considered meaningful [31]. For the purpose of these analyses, standardized difference was calculated as the difference in mean (or proportion for binary variables) divided by the standard deviation (pooled standard deviation for the continuous variables). Conditional logistic regression was used to determine adjusted odds ratios with $95 \%$ confidence intervals of saxagliptin use associated with demographic variables, comorbidities, and drug therapies. To explore whether the determinants of saxagliptin use were different between first-time OAD initiators and those who were previously treated with OADs, we re-ran analyses in each database, stratified by whether patients received prior OAD therapy. Because the conditional logistic regression is conditioned on the matched group, initiators who no longer had a matched comparator in the stratified cohorts were removed from this analysis. Data were analyzed using SAS 9.4 (SAS Institute Inc., Cary, NC). 
Table 1 Demographic characteristics of type 2 diabetes mellitus patients within United Kingdom data sources

\begin{tabular}{|c|c|c|c|c|c|c|}
\hline \multirow{3}{*}{ Characteristic $^{*}$} & \multicolumn{3}{|c|}{ Clinical Practice Research Datalink } & \multicolumn{3}{|c|}{ The Health Improvement Network } \\
\hline & Saxagliptin & $\begin{array}{l}\text { Other } \\
\text { OAD }\end{array}$ & $\begin{array}{l}\text { Standardized } \\
\text { difference }\end{array}$ & Saxagliptin & $\begin{array}{l}\text { Other } \\
\text { OAD }\end{array}$ & $\begin{array}{l}\text { Standardized } \\
\text { difference }\end{array}$ \\
\hline & $(n=1,962)$ & $(n=19,484)$ & & $(n=2,084)$ & $(n=19,936)$ & \\
\hline Mean (SD) age, years ${ }^{\dagger}$ & $52.7(10.6)$ & $52.2(10.6)$ & 0.01 & $64.7(12.9)$ & $64.6(12.9)$ & 0.01 \\
\hline Male sex ${ }^{\dagger}$ & $58.2 \%$ & $58.2 \%$ & $<0.01$ & $57.7 \%$ & $57.0 \%$ & 0.01 \\
\hline \multicolumn{7}{|l|}{ UK country $^{\dagger}$} \\
\hline England & $60.4 \%$ & $60.6 \%$ & $<0.01$ & $63.1 \%$ & $65.7 \%$ & 0.05 \\
\hline Northern Ireland & $7.1 \%$ & $7.0 \%$ & $<0.01$ & $5.3 \%$ & $5.0 \%$ & 0.01 \\
\hline Scotland & $11.4 \%$ & $11.5 \%$ & $<0.01$ & $12.3 \%$ & $12.8 \%$ & 0.01 \\
\hline Wales & $21.0 \%$ & $20.9 \%$ & $<0.01$ & $19.2 \%$ & $16.5 \%$ & 0.07 \\
\hline \multicolumn{7}{|l|}{ Other OAD initiated at index date } \\
\hline Alpha-glucosidase inhibitors: Acarbose & $0 \%$ & $0.2 \%$ & - & $0 \%$ & $0.2 \%$ & - \\
\hline Biguanide: Metformin & $0 \%$ & $63.1 \%$ & - & $0 \%$ & $62.1 \%$ & - \\
\hline Meglitinides & $0 \%$ & $0.4 \%$ & - & $0 \%$ & $0.4 \%$ & - \\
\hline Nateglinide & $0 \%$ & $0.1 \%$ & - & $0 \%$ & $0.0 \%$ & - \\
\hline Repaglinide & $0 \%$ & $0.3 \%$ & - & $0 \%$ & $0.3 \%$ & - \\
\hline Sulfonylureas & $0 \%$ & $27.9 \%$ & - & $0 \%$ & $29.1 \%$ & - \\
\hline Glibenclamide (Glyburide in US data sources) & $0 \%$ & $0.2 \%$ & - & $0 \%$ & $0.2 \%$ & - \\
\hline Gliclazide & $0 \%$ & $24.9 \%$ & - & $0 \%$ & $26.1 \%$ & - \\
\hline Glimepiride & $0 \%$ & $2.1 \%$ & - & $0 \%$ & $1.9 \%$ & - \\
\hline Glipizide & $0 \%$ & $0.6 \%$ & - & $0 \%$ & $0.7 \%$ & - \\
\hline Tolbutamide & $0 \%$ & $0.1 \%$ & - & $0 \%$ & $0.2 \%$ & - \\
\hline Thiazolidinediones & $0 \%$ & $8.3 \%$ & - & $0 \%$ & $8.2 \%$ & - \\
\hline Pioglitazone & $0 \%$ & $8.1 \%$ & - & $0 \%$ & $8.0 \%$ & - \\
\hline Rosiglitazone & $0 \%$ & $0.2 \%$ & - & $0 \%$ & $0.2 \%$ & - \\
\hline On glucagon-like peptide-1 receptor agonist & $3.1 \%$ & $1.9 \%$ & 0.08 & $2.4 \%$ & $1.9 \%$ & 0.03 \\
\hline On insulin & $5.4 \%$ & $7.3 \%$ & 0.08 & $7.7 \%$ & $7.2 \%$ & 0.02 \\
\hline \multicolumn{7}{|l|}{ Hemoglobin A1c measurements } \\
\hline Mean (SD) & $8.7(1.6)$ & $8.6(1.8)$ & 0.06 & $8.8(1.6)$ & $8.6(1.8)$ & 0.08 \\
\hline Hemoglobin A1c $>8 \%$ & $57.6 \%$ & $39.8 \%$ & 0.36 & $56.2 \%$ & $40.8 \%$ & 0.31 \\
\hline Mean body mass index (SD) & $32.4(6.5)$ & $31.5(6.7)$ & 0.14 & - & - & - \\
\hline Missing values & $42.9 \%$ & $41.4 \%$ & 0.03 & $0.7 \%$ & $2.0 \%$ & 0.11 \\
\hline Underweight $\left(15-18.5 \mathrm{~kg} / \mathrm{m}^{2}\right)$ & $0.2 \%$ & $0.4 \%$ & 0.03 & $0.0 \%$ & $0.4 \%$ & 0.08 \\
\hline Normal $\left(18.5-24.9 \mathrm{~kg} / \mathrm{m}^{2}\right)$ & $4.4 \%$ & $6.7 \%$ & 0.10 & $7.9 \%$ & $11.4 \%$ & 0.12 \\
\hline Overweight $(25.0-29.9$ kg/m²) & $15.9 \%$ & $17.8 \%$ & 0.05 & $29.0 \%$ & $31.5 \%$ & 0.05 \\
\hline Obese $(30-60$ kg/m²) & $36.7 \%$ & $33.9 \%$ & 0.06 & $62.3 \%$ & $54.7 \%$ & 0.15 \\
\hline Smoking & $36.5 \%$ & $36.5 \%$ & $<0.01$ & $63.5 \%$ & $61.0 \%$ & 0.05 \\
\hline \multicolumn{7}{|l|}{ Severity of type 2 diabetes mellitus (prior $180 \mathrm{~d}$ ) } \\
\hline Cerebrovascular disease & $0.5 \%$ & $0.7 \%$ & 0.02 & $0.9 \%$ & $0.7 \%$ & 0.02 \\
\hline $\begin{array}{l}\text { Coronary artery disease, congestive heart failure, } \\
\text { ventricular tachycardia/fibrillation }\end{array}$ & $1.3 \%$ & $1.5 \%$ & 0.01 & $1.9 \%$ & $1.5 \%$ & 0.03 \\
\hline Diabetic coma & $0 \%$ & $0.1 \%$ & - & $0 \%$ & $0.1 \%$ & - \\
\hline Nephropathy & $0.3 \%$ & $0.2 \%$ & 0.02 & $0.6 \%$ & $0.2 \%$ & 0.06 \\
\hline Neuropathy & $0.8 \%$ & $0.6 \%$ & 0.02 & $0.9 \%$ & $0.6 \%$ & 0.03 \\
\hline Peripheral vascular disease & $1.1 \%$ & $0.9 \%$ & 0.02 & $1.2 \%$ & $0.9 \%$ & 0.03 \\
\hline
\end{tabular}


Table 1 Demographic characteristics of type 2 diabetes mellitus patients within United Kingdom data sources (Continued)

\begin{tabular}{|c|c|c|c|c|c|c|}
\hline Retinopathy & $5.7 \%$ & $3.7 \%$ & 0.09 & $5.5 \%$ & $3.5 \%$ & 0.10 \\
\hline Unspecified additional diabetic complications & $0 \%$ & $0.0 \%$ & - & $0 \%$ & $0.0 \%$ & - \\
\hline \multicolumn{7}{|l|}{ Medical comorbidities } \\
\hline Allergic rhinitis/hay fever & $8.8 \%$ & $10.0 \%$ & 0.04 & $9.1 \%$ & $9.4 \%$ & 0.01 \\
\hline Asthma & $16.0 \%$ & $15.8 \%$ & $<0.01$ & $16.4 \%$ & $15.9 \%$ & 0.01 \\
\hline Chronic obstructive pulmonary disease/bronchitis & $12.1 \%$ & $10.0 \%$ & 0.07 & $13.1 \%$ & $10.7 \%$ & 0.07 \\
\hline \multicolumn{7}{|l|}{ Dermatologic disorder } \\
\hline Eczema & $17.0 \%$ & $15.2 \%$ & 0.05 & $16.9 \%$ & $14.2 \%$ & 0.08 \\
\hline Psoriasis/psoriatic arthritis & $6.4 \%$ & $5.1 \%$ & 0.06 & $5.8 \%$ & $5.3 \%$ & 0.02 \\
\hline \multicolumn{7}{|l|}{ Gastrointestinal disease } \\
\hline Cirrhosis & $0.3 \%$ & $0.4 \%$ & 0.01 & $0.2 \%$ & $0.4 \%$ & 0.03 \\
\hline Gallbladder disease & $6.2 \%$ & $5.6 \%$ & 0.02 & $6.3 \%$ & $5.9 \%$ & 0.02 \\
\hline Hemochromatosis & $0.1 \%$ & $0.1 \%$ & 0.02 & $0 \%$ & $0.2 \%$ & 0.04 \\
\hline Hyperlipidemia & $16.0 \%$ & $12.0 \%$ & 0.12 & $15.9 \%$ & $12.2 \%$ & 0.11 \\
\hline Hypertension & $56.7 \%$ & $50.9 \%$ & 0.12 & $61.0 \%$ & $56.1 \%$ & 0.10 \\
\hline \multicolumn{7}{|l|}{ Infectious disease } \\
\hline Hepatitis B virus infection & $0.3 \%$ & $0.2 \%$ & 0.02 & $0.2 \%$ & $0.2 \%$ & 0.01 \\
\hline Hepatitis C virus infection & $0 \%$ & $0.1 \%$ & - & $0 \%$ & $0.1 \%$ & - \\
\hline \multicolumn{7}{|l|}{ Malignancy } \\
\hline Hematologic & $0.8 \%$ & $1.0 \%$ & 0.03 & $0.9 \%$ & $1.0 \%$ & $<0.01$ \\
\hline Solid organ & $23.5 \%$ & $22.9 \%$ & 0.02 & $22.9 \%$ & $23.9 \%$ & 0.02 \\
\hline Obesity & $17.8 \%$ & $14.6 \%$ & 0.09 & $17.0 \%$ & $13.4 \%$ & 0.10 \\
\hline Rheumatoid arthritis & $2.7 \%$ & $2.4 \%$ & 0.02 & $1.3 \%$ & $1.7 \%$ & 0.03 \\
\hline \multicolumn{7}{|l|}{ Medications } \\
\hline Acetaminophen/paracetamol & $31.7 \%$ & $28.7 \%$ & 0.06 & $32.0 \%$ & $30.1 \%$ & 0.04 \\
\hline Anti-asthmatic agents & $18.6 \%$ & $17.7 \%$ & 0.02 & $18.8 \%$ & $18.1 \%$ & 0.02 \\
\hline Antibacterials & $35.5 \%$ & $33.9 \%$ & 0.03 & $32.1 \%$ & $30.7 \%$ & 0.03 \\
\hline Anticonvulsants & $8.8 \%$ & $7.8 \%$ & 0.04 & $6.2 \%$ & $5.1 \%$ & 0.05 \\
\hline Antifungals & $3.0 \%$ & $2.6 \%$ & 0.02 & $2.9 \%$ & $3.4 \%$ & 0.03 \\
\hline Antihistamines & $7.2 \%$ & $7.1 \%$ & $<0.01$ & $7.5 \%$ & $6.9 \%$ & 0.02 \\
\hline Anti-hyperlipidemic agents & $80.6 \%$ & $58.5 \%$ & 0.49 & $81.1 \%$ & $61.9 \%$ & 0.43 \\
\hline \multicolumn{7}{|l|}{ Antihypertensive agents } \\
\hline Angiotensin-converting enzyme inhibitors & $46.5 \%$ & $36.1 \%$ & 0.21 & $46.1 \%$ & $38.4 \%$ & 0.16 \\
\hline Angiotensin receptor blockers & $19.2 \%$ & $13.2 \%$ & 0.16 & $22.5 \%$ & $15.0 \%$ & 0.19 \\
\hline Beta blockers & $24.7 \%$ & $20.7 \%$ & 0.09 & $28.0 \%$ & $23.2 \%$ & 0.11 \\
\hline Calcium channel blockers & $28.2 \%$ & $23.8 \%$ & 0.10 & $29.9 \%$ & $26.9 \%$ & 0.07 \\
\hline Loop diuretics & $12.4 \%$ & $11.0 \%$ & 0.04 & $17.3 \%$ & $11.4 \%$ & 0.17 \\
\hline Other antihypertensive agents & $9.2 \%$ & $6.8 \%$ & 0.09 & $10.0 \%$ & $6.8 \%$ & 0.11 \\
\hline Thiazide diuretics & $19.9 \%$ & $16.1 \%$ & 0.10 & $25.1 \%$ & $20.8 \%$ & 0.10 \\
\hline Antivirals & $0.7 \%$ & $0.8 \%$ & 0.02 & $0.8 \%$ & $0.9 \%$ & 0.02 \\
\hline Non-aspirin non-steroidal anti-inflammatory & $13.4 \%$ & $13.0 \%$ & 0.01 & $12.5 \%$ & $13.0 \%$ & 0.02 \\
\hline \multicolumn{7}{|l|}{ Other antiplatelet/anticoagulant agents } \\
\hline Aspirin & $38.3 \%$ & $29.7 \%$ & 0.18 & $42.1 \%$ & $32.2 \%$ & 0.21 \\
\hline Clopidogrel & $4.9 \%$ & $3.7 \%$ & 0.06 & $5.0 \%$ & $3.7 \%$ & 0.06 \\
\hline
\end{tabular}


Table 1 Demographic characteristics of type 2 diabetes mellitus patients within United Kingdom data sources (Continued)

\begin{tabular}{|c|c|c|c|c|c|c|}
\hline Low-molecular-weight heparin & $0.2 \%$ & $0.3 \%$ & 0.03 & $0.5 \%$ & $0.3 \%$ & 0.02 \\
\hline Warfarin & $5.4 \%$ & $4.9 \%$ & 0.02 & $6.9 \%$ & $5.6 \%$ & 0.05 \\
\hline \multicolumn{7}{|l|}{ Other medications } \\
\hline Allopurinol & $3.2 \%$ & $3.5 \%$ & 0.02 & $5.1 \%$ & $3.7 \%$ & 0.07 \\
\hline Anti-arrhythmics & $3.4 \%$ & $2.6 \%$ & 0.05 & $3.1 \%$ & $3.0 \%$ & $<0.01$ \\
\hline Immune modulators/immunosuppressants & $1.2 \%$ & $1.2 \%$ & $<0.01$ & $1.2 \%$ & $1.2 \%$ & 0.01 \\
\hline Nitroglycerin & $5.6 \%$ & $5.1 \%$ & 0.02 & $6.3 \%$ & $4.9 \%$ & 0.06 \\
\hline Urinary anti-spasmodics & $4.3 \%$ & $3.3 \%$ & 0.05 & $4.7 \%$ & $3.6 \%$ & 0.06 \\
\hline \multicolumn{7}{|l|}{ Psychotropic agents } \\
\hline Antidepressants & $22.1 \%$ & $19.6 \%$ & 0.06 & $20.6 \%$ & $19.6 \%$ & 0.03 \\
\hline Antipsychotics & $4.3 \%$ & $4.4 \%$ & 0.01 & $4.9 \%$ & $4.8 \%$ & 0.01 \\
\hline Prior OAD Therapy ${ }^{\ddagger}$ & $93.7 \%$ & $35.8 \%$ & 1.53 & $92.1 \%$ & $36.8 \%$ & 1.41 \\
\hline Alpha-glucosidase inhibitors: Acarbose & $0.3 \%$ & $0.1 \%$ & 0.03 & $0.2 \%$ & $0.1 \%$ & 0.03 \\
\hline Biguanide: Metformin & $83.4 \%$ & $73.7 \%$ & 0.24 & $77.7 \%$ & $73.1 \%$ & 0.11 \\
\hline Meglitinides & $1.1 \%$ & $0.4 \%$ & 0.09 & $1.2 \%$ & $0.3 \%$ & 0.10 \\
\hline Nateglinide & $0.2 \%$ & $0.0 \%$ & 0.04 & $0.2 \%$ & $0.1 \%$ & 0.04 \\
\hline Repaglinide & $0.9 \%$ & $0.3 \%$ & 0.08 & $1.0 \%$ & $0.2 \%$ & 0.10 \\
\hline Sulfonylureas & $47.9 \%$ & $12.2 \%$ & 0.84 & $46.4 \%$ & $12.8 \%$ & 0.79 \\
\hline Glibenclamide & $0.4 \%$ & $0.5 \%$ & 0.01 & $0.5 \%$ & $0.6 \%$ & 0.01 \\
\hline Gliclazide & $41.4 \%$ & $12.6 \%$ & 0.69 & $39.3 \%$ & $13.4 \%$ & 0.61 \\
\hline Glimepiride & $4.5 \%$ & $1.5 \%$ & 0.18 & $4.1 \%$ & $1.4 \%$ & 0.17 \\
\hline Glipizide & $1.1 \%$ & $0.7 \%$ & 0.04 & $1.7 \%$ & $0.8 \%$ & 0.09 \\
\hline Tolbutamide & $0.7 \%$ & $0.1 \%$ & 0.09 & $1.1 \%$ & $0.2 \%$ & 0.11 \\
\hline Thiazolidinediones & $16.6 \%$ & $5.3 \%$ & 0.37 & $17.7 \%$ & $5.1 \%$ & 0.40 \\
\hline Pioglitazone & $14.1 \%$ & $3.5 \%$ & 0.38 & $15.4 \%$ & $3.4 \%$ & 0.42 \\
\hline Rosiglitazone & $2.7 \%$ & $2.1 \%$ & 0.03 & $2.5 \%$ & $2.0 \%$ & 0.03 \\
\hline
\end{tabular}

Abbreviations: $\mathrm{OAD}=$ oral anti-diabetic drug; $\mathrm{SD}=$ standard deviation.

${ }^{*}$ Characteristics are presented as percentages unless otherwise indicated.

${ }^{\dagger}$ Matching criteria for which a random sample (without replacement) of up to ten new initiators of non-DPP-4 inhibitor OADs were selected for each saxagliptin initiator.

${ }^{\ddagger}$ Defined as use of an oral anti-diabetic drug within the 180 days prior to the initiation of the index drug. Denominator adjusted to exclude those on index drug.

\section{Results}

\section{Patient characteristics}

\section{UK data sources}

Within THIN and CPRD, respectively, we identified, 1,962 and 2,084 new initiators of saxagliptin (Figure 1a and $\mathrm{b}$ ) as well as 19,484 and 19,936 matched new initiators of non-DPP-4 inhibitor OADs. The characteristics of these patients are presented in Table 1. Approximately 6\% of saxagliptin initiators in each UK data source had not received treatment for T2DM with another OAD within 180 days prior to the index date.

Within both UK data sources, saxagliptin initiators were more likely to have had prior OAD therapy and hemoglobin A1c results $>8 \%$ in the 180 days preceding the index prescription compared to initiators of other non-DPP-4 inhibitor OADs. Saxagliptin users also more frequently had hyperlipidemia, hypertension, and obesity and were more commonly prescribed aspirin, anti-hyperlipidemic agents, and anti-hypertensive drugs. Within THIN, diagnoses of retinopathy were more prevalent among saxagliptin initiators.

\section{US data sources}

During the initial 29 months of saxagliptin availability within US Medicare, 51,976 new initiators of saxagliptin and 493,432 matched new initiators of non-DPP-4 inhibitor OADs were identified (Figure 1c). During the initial 36 months of saxagliptin availability within the HIRD ${ }^{\text {SM }}, 8,057$ new initiators of saxagliptin and 77,808 matched new initiators of non-DPP-4 inhibitor OADs were identified (Figure 1d). The characteristics of these patients are presented in Table 2. Approximately $22 \%$ of saxagliptin initiators in US Medicare and $33 \%$ of saxagliptin initiators in the HIRD ${ }^{\mathrm{SM}}$ had not 

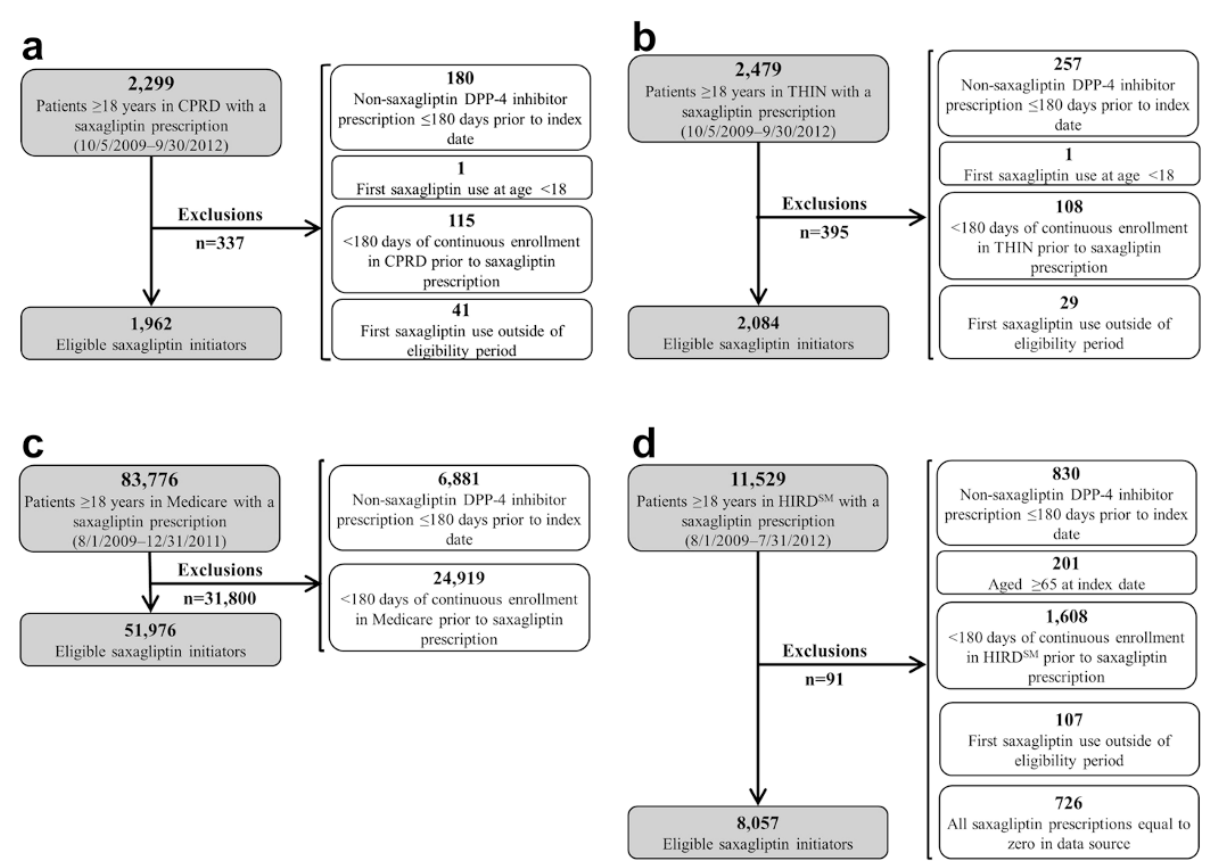

Figure 1 Selection of saxagliptin patients. a: Clinical Practice Research Datalink (CPRD). b: The Health Improvement Network (THIN). c: US Medicare. d: HealthCore Integrated Research Database ${ }^{S M}\left(H_{R} D^{S M}\right)$.

received treatment for T2DM with another $\mathrm{OAD}$ within 180 days prior to the index date.

Within both US databases, saxagliptin initiators more frequently received prior OAD therapy and had higher mean numbers of hemoglobin A1c measurements in the 180 days prior to the index date. Saxagliptin initiators were also more frequently diagnosed with hyperlipidemia and hypertension and more commonly received anti-hyperlipidemic drugs, angiotensinconverting enzyme inhibitors, and angiotensin receptor blockers. Within US Medicare, diagnoses of microvascular T2DM complications, including nephropathy and neuropathy, were more prevalent among saxagliptin initiators.

\section{Factors associated with saxagliptin use \\ UK data sources}

Factors associated with saxagliptin initiation within CPRD and THIN are presented in Table 3. Prior OAD use in the 180 days preceding the index date was strongly associated with saxagliptin initiation in CPRD and THIN. Within THIN, diabetic nephropathy and obesity were also associated with a higher likelihood of saxagliptin initiation.

After stratifying on prior OAD use within CPRD, results were similar to those in the primary analysis. However, prescriptions for antihyperlipidemic agents and diagnoses of chronic obstructive pulmonary disease and bronchitis were associated with saxagliptin initiation among patients without prior OAD use (Table 3).

After stratifying on prior OAD use within THIN, results were also similar to those in the primary analysis. However, obesity and diabetic nephropathy were more strongly associated with saxagliptin initiation among those with prior OAD use. Additionally, among patients with no prior OAD use, prescriptions for antihyperlipidemics and diagnoses of retinopathy were associated with saxagliptin initiation (Table 3).

\section{US data sources}

Factors associated with saxagliptin initiation in US Medicare and the HIRD ${ }^{\mathrm{SM}}$ are presented in Table 4. Saxagliptin initiation was associated with prior OAD use and a greater number of hemoglobin A1c measurements in the 180 days preceding the index date in Medicare and the HIRD $^{\text {SM }}$. Additionally, within Medicare, saxagliptin initiators were more likely to receive angiotensin-receptor blockers.

After stratifying on prior OAD use within US Medicare, results were similar to those in the primary analysis. However, use of angiotensin-receptor blockers was more strongly associated with saxagliptin use among those without prior OAD use (Table 4).

After stratifying on prior OAD use within the HIRD $^{\mathrm{SM}}$, results remained similar to those within the primary analyses (Table 4). 
Table 2 Demographic characteristics of type 2 diabetes mellitus patients within United States data sources

\begin{tabular}{|c|c|c|c|c|c|c|}
\hline \multirow{3}{*}{ Characteristic $^{*}$} & \multicolumn{3}{|l|}{ US Medicare } & \multicolumn{3}{|c|}{$\begin{array}{l}\text { HealthCore Integrated Reseach } \\
\text { Database }\end{array}$} \\
\hline & Saxagliptin & $\begin{array}{l}\text { Other } \\
\text { OAD }\end{array}$ & $\begin{array}{l}\text { Standardized } \\
\text { difference }\end{array}$ & Saxagliptin & $\begin{array}{l}\text { Other } \\
\text { OAD }\end{array}$ & $\begin{array}{l}\text { Standardized } \\
\text { difference }\end{array}$ \\
\hline & $(n=51,976)$ & $(n=493,432)$ & & $(n=8,057)$ & $(\mathrm{n}=77,808)$ & \\
\hline Mean (SD) age, years ${ }^{\dagger}$ & $70.4(11.1)$ & $69.9(11.0)$ & 0.04 & $52.9(8.4)$ & $52.9(8.4)$ & 0.01 \\
\hline Male sex ${ }^{\dagger}$ & $42.1 \%$ & $42.8 \%$ & 0.01 & $60.0 \%$ & $59.5 \%$ & 0.01 \\
\hline \multicolumn{7}{|l|}{ US census region $^{\dagger}$} \\
\hline East North Central & $12.7 \%$ & $12.8 \%$ & $<0.01$ & $20.7 \%$ & $21.2 \%$ & 0.01 \\
\hline East South Central & $10.0 \%$ & $10.0 \%$ & $<0.01$ & $10.0 \%$ & $10.1 \%$ & $<0.01$ \\
\hline Middle Atlantic & $16.0 \%$ & $15.9 \%$ & $<0.01$ & $9.0 \%$ & $8.9 \%$ & $<0.01$ \\
\hline Mountain & $3.1 \%$ & $3.1 \%$ & $<0.01$ & $1.9 \%$ & $1.9 \%$ & $<0.01$ \\
\hline New England & $2.8 \%$ & $2.8 \%$ & $<0.01$ & $5.7 \%$ & $5.9 \%$ & $<0.01$ \\
\hline Pacific & $12.8 \%$ & $13.1 \%$ & $<0.01$ & $14.6 \%$ & $14.8 \%$ & $<0.01$ \\
\hline South Atlantic & $24.1 \%$ & $24.0 \%$ & $<0.01$ & $30.5 \%$ & $29.9 \%$ & 0.01 \\
\hline West North Central & $5.2 \%$ & $5.0 \%$ & $<0.01$ & $5.3 \%$ & $5.4 \%$ & $<0.01$ \\
\hline West South Central & $13.2 \%$ & $13.2 \%$ & $<0.01$ & $2.2 \%$ & $1.9 \%$ & 0.02 \\
\hline \multicolumn{7}{|l|}{ Other OAD initiated at index date } \\
\hline Alpha-glucosidase inhibitors & $0 \%$ & $0.6 \%$ & - & $0 \%$ & $0.4 \%$ & - \\
\hline Acarbose & $0 \%$ & $0.5 \%$ & - & $0 \%$ & $0.3 \%$ & - \\
\hline Miglitol & $0 \%$ & $0.0 \%$ & - & $0 \%$ & $0.0 \%$ & - \\
\hline Biguanide: Metformin & $0 \%$ & $51.5 \%$ & - & $0 \%$ & $69.1 \%$ & - \\
\hline Meglitinides & $0 \%$ & $2.4 \%$ & - & $0 \%$ & $0.9 \%$ & - \\
\hline Nateglinide & $0 \%$ & $1.0 \%$ & - & $0 \%$ & $0.4 \%$ & - \\
\hline Repaglinide & $0 \%$ & $1.3 \%$ & - & $0 \%$ & $0.5 \%$ & - \\
\hline Sulfonylureas & $0 \%$ & $33.8 \%$ & - & $0 \%$ & $22.0 \%$ & - \\
\hline Chlorpropamide & $0 \%$ & $0.0 \%$ & - & $0 \%$ & $0.0 \%$ & - \\
\hline Glimepiride & $0 \%$ & $11.0 \%$ & - & $0 \%$ & $8.1 \%$ & - \\
\hline Glipizide & $0 \%$ & $14.3 \%$ & - & $0 \%$ & $8.3 \%$ & - \\
\hline Glyburide (glibenclamide in UK data sources) & $0 \%$ & $8.5 \%$ & - & $0 \%$ & $5.6 \%$ & - \\
\hline Tolazamide & $0 \%$ & $0.0 \%$ & - & $0 \%$ & $0.0 \%$ & - \\
\hline Tolbutamide & $0 \%$ & $0.0 \%$ & - & $0 \%$ & $0.0 \%$ & - \\
\hline Thiazolidinediones & $0 \%$ & $11.7 \%$ & - & $0 \%$ & $7.7 \%$ & - \\
\hline Pioglitazone & $0 \%$ & $11.0 \%$ & - & $0 \%$ & $7.3 \%$ & - \\
\hline Rosiglitazone & $0 \%$ & $0.8 \%$ & - & $0 \%$ & $0.4 \%$ & - \\
\hline On glucagon-like peptide- 1 receptor agonist & $2.1 \%$ & $1.1 \%$ & 0.07 & $3.8 \%$ & $2.9 \%$ & 0.05 \\
\hline On insulin & $15.9 \%$ & $14.9 \%$ & 0.03 & $9.7 \%$ & $8.3 \%$ & 0.05 \\
\hline Mean (SD) number of hemoglobin A1c measures & $1.3(0.9)$ & $0.9(0.9)$ & 0.51 & $1.1(0.8)$ & $0.8(0.8)$ & 0.50 \\
\hline \multicolumn{7}{|l|}{ Severity of type 2 diabetes mellitus (prior $180 \mathrm{~d}$ ) } \\
\hline Cerebrovascular disease & $10.5 \%$ & $10.6 \%$ & $<0.01$ & $2.4 \%$ & $2.4 \%$ & $<0.01$ \\
\hline $\begin{array}{l}\text { Coronary artery disease, congestive heart failure, ventricular } \\
\text { tachycardia/fibrillation }\end{array}$ & $40.8 \%$ & $38.2 \%$ & 0.05 & $11.8 \%$ & $11.1 \%$ & 0.02 \\
\hline Metabolic (ketoacidosis, hyperosmolar,coma) & $1.3 \%$ & $1.3 \%$ & $<0.01$ & $0.7 \%$ & $0.7 \%$ & $<0.01$ \\
\hline Nephropathy & $20.2 \%$ & $15.7 \%$ & 0.11 & $5.0 \%$ & $3.6 \%$ & 0.07 \\
\hline Neuropathy & $22.9 \%$ & $18.1 \%$ & 0.12 & $8.3 \%$ & $6.8 \%$ & 0.06 \\
\hline Peripheral vascular disease & $18.4 \%$ & $16.6 \%$ & 0.05 & $3.9 \%$ & $3.4 \%$ & 0.03 \\
\hline
\end{tabular}


Table 2 Demographic characteristics of type 2 diabetes mellitus patients within United States data sources (Continued)

\begin{tabular}{|c|c|c|c|c|c|c|}
\hline Retinopathy & $13.3 \%$ & $10.5 \%$ & 0.09 & $5.3 \%$ & $3.9 \%$ & 0.07 \\
\hline Unspecified additional diabetic complications & $7.9 \%$ & $6.9 \%$ & 0.04 & $3.8 \%$ & $2.7 \%$ & 0.06 \\
\hline \multicolumn{7}{|l|}{ Medical comorbidities } \\
\hline Allergic rhinitis/hay fever & $7.1 \%$ & $5.6 \%$ & 0.06 & $5.2 \%$ & $4.6 \%$ & 0.03 \\
\hline Asthma & $7.7 \%$ & $7.9 \%$ & 0.01 & $3.8 \%$ & $4.3 \%$ & 0.02 \\
\hline Chronic obstructive pulmonary disease/bronchitis & $12.5 \%$ & $13.4 \%$ & 0.03 & $2.7 \%$ & $3.0 \%$ & 0.02 \\
\hline \multicolumn{7}{|l|}{ Dermatologic disorders } \\
\hline Eczema & $3.5 \%$ & $3.0 \%$ & 0.02 & $2.2 \%$ & $1.9 \%$ & 0.02 \\
\hline Psoriasis/psoriatic arthritis & $0.9 \%$ & $0.9 \%$ & $<0.01$ & $0.9 \%$ & $0.9 \%$ & 0.01 \\
\hline \multicolumn{7}{|l|}{ Gastrointestinal disease } \\
\hline Cirrhosis & $0.8 \%$ & $0.8 \%$ & $<0.01$ & $0.3 \%$ & $0.4 \%$ & 0.01 \\
\hline Gallbladder disease & $2.2 \%$ & $2.3 \%$ & 0.01 & $1.1 \%$ & $1.1 \%$ & $<0.01$ \\
\hline Hemochromatosis & $0.3 \%$ & $0.2 \%$ & 0.01 & $0.2 \%$ & $0.2 \%$ & $<0.01$ \\
\hline Hyperlipidemia & $77.1 \%$ & $66.3 \%$ & 0.24 & $62.0 \%$ & $48.7 \%$ & 0.27 \\
\hline Hypertension & $85.3 \%$ & $78.1 \%$ & 0.18 & $60.9 \%$ & $50.9 \%$ & 0.20 \\
\hline \multicolumn{7}{|l|}{ Infections } \\
\hline Hepatitis B virus infection & $0.2 \%$ & $0.2 \%$ & $<0.01$ & $0.2 \%$ & $0.2 \%$ & 0.01 \\
\hline Hepatitis C virus infection & $0.8 \%$ & $0.9 \%$ & 0.02 & $0.5 \%$ & $0.5 \%$ & $<0.01$ \\
\hline Human immunodeficiency virus & $0.2 \%$ & $0.4 \%$ & 0.02 & $0.1 \%$ & $0.2 \%$ & 0.03 \\
\hline \multicolumn{7}{|l|}{ Malignancy } \\
\hline Hematologic & $1.3 \%$ & $1.3 \%$ & 0.01 & $0.6 \%$ & $0.6 \%$ & 0.01 \\
\hline Solid organ & $8.2 \%$ & $8.6 \%$ & 0.01 & $3.3 \%$ & $3.3 \%$ & $<0.01$ \\
\hline Obesity & $11.1 \%$ & $10.7 \%$ & 0.01 & $9.3 \%$ & $9.4 \%$ & $<0.01$ \\
\hline Rheumatoid arthritis & $2.8 \%$ & $2.5 \%$ & 0.02 & $0.9 \%$ & $0.9 \%$ & $<0.01$ \\
\hline \multicolumn{7}{|l|}{ Medications } \\
\hline Acetaminophen/paracetamol & $28.1 \%$ & $26.3 \%$ & 0.04 & $20.2 \%$ & $19.8 \%$ & 0.01 \\
\hline Anti-asthmatic agents & $14.4 \%$ & $12.5 \%$ & 0.05 & $8.3 \%$ & $7.9 \%$ & 0.01 \\
\hline Antibacterials & $46.3 \%$ & $40.3 \%$ & 0.12 & $38.1 \%$ & $35.7 \%$ & 0.05 \\
\hline Anticonvulsants & $5.4 \%$ & $5.6 \%$ & 0.01 & $7.2 \%$ & $7.5 \%$ & 0.01 \\
\hline Antifungals & $10.1 \%$ & $8.1 \%$ & 0.07 & $6.8 \%$ & $5.7 \%$ & 0.05 \\
\hline Antihistamines & $11.8 \%$ & $9.4 \%$ & 0.08 & $6.4 \%$ & $5.7 \%$ & 0.03 \\
\hline Anti-hyperlipidemic agents & $69.2 \%$ & $52.8 \%$ & 0.34 & $53.5 \%$ & $38.6 \%)$ & 0.30 \\
\hline \multicolumn{7}{|l|}{ Antihypertensive agents } \\
\hline Angiotensin-converting enzyme inhibitors & $43.1 \%$ & $37.2 \%$ & 0.12 & $35.8 \%$ & $28.0 \%$ & 0.17 \\
\hline Angiotensin receptor blockers & $28.5 \%$ & $18.0 \%$ & 0.25 & $20.1 \%$ & $14.1 \%$ & 0.16 \\
\hline Beta blockers & $44.0 \%$ & $36.1 \%$ & 0.16 & $21.2 \%$ & $18.4 \%$ & 0.07 \\
\hline Calcium channel blockers & $31.7 \%$ & $26.3 \%$ & 0.12 & $16.0 \%$ & $13.9 \%$ & 0.06 \\
\hline Loop diuretics & $23.1 \%$ & $18.1 \%$ & 0.12 & $5.1 \%$ & $4.7 \%$ & 0.02 \\
\hline Other antihypertensive agents & $11.9 \%$ & $9.0 \%$ & 0.10 & $4.8 \%$ & $3.9 \%$ & 0.04 \\
\hline Thiazide diuretics & $21.7 \%$ & $15.6 \%$ & 0.16 & $18.2 \%$ & $15.0 \%$ & 0.09 \\
\hline Antivirals & $2.3 \%$ & $2.0 \%$ & 0.02 & $1.9 \%$ & $2.5 \%$ & 0.04 \\
\hline Non-aspirin non-steroidal anti-inflammatory & $17.4 \%$ & $14.7 \%$ & 0.08 & $13.9 \%$ & $12.9 \%$ & 0.03 \\
\hline \multicolumn{7}{|l|}{ Other antiplatelet/anticoagulant agents } \\
\hline Aspirin & $0.8 \%$ & $0.6 \%$ & 0.02 & $0.1 \%$ & $0.1 \%$ & 0.01 \\
\hline Clopidogrel & $13.7 \%$ & $10.4 \%$ & 0.10 & $4.0 \%$ & $3.6 \%$ & 0.02 \\
\hline
\end{tabular}


Table 2 Demographic characteristics of type 2 diabetes mellitus patients within United States data sources (Continued)

\begin{tabular}{|c|c|c|c|c|c|c|}
\hline Low-molecular-weight heparin & $0.5 \%$ & $0.7 \%$ & 0.02 & $0.4 \%$ & $0.4 \%$ & 0.01 \\
\hline Warfarin & $7.4 \%$ & $7.0 \%$ & 0.02 & $2.1 \%$ & $1.7 \%$ & 0.03 \\
\hline \multicolumn{7}{|l|}{ Other medications } \\
\hline Allopurinol & $5.4 \%$ & $4.1 \%$ & 0.06 & $2.5 \%$ & $2.4 \%$ & $<0.01$ \\
\hline Anti-arrhythmics & $13.6 \%$ & $12.2 \%$ & 0.04 & $4.7 \%$ & $4.6 \%$ & 0.01 \\
\hline Immune modulators/immunosuppressants & $4.5 \%$ & $4.1 \%$ & 0.02 & $2.2 \%$ & $2.4 \%$ & 0.01 \\
\hline Nitroglycerin & $4.6 \%$ & $3.8 \%$ & 0.04 & $1.3 \%$ & $1.3 \%$ & $<0.01$ \\
\hline Urinary anti-spasmodics & $5.4 \%$ & $4.5 \%$ & 0.04 & $0.9 \%$ & $1.2 \%$ & 0.03 \\
\hline \multicolumn{7}{|l|}{ Psychotropic agents } \\
\hline Antidepressants & $27.4 \%$ & $25.3 \%$ & 0.05 & $19.1 \%$ & $18.9 \%$ & $<0.01$ \\
\hline Antipsychotics & $7.5 \%$ & $7.8 \%$ & 0.01 & $2.1 \%$ & $2.3 \%$ & 0.01 \\
\hline Prior OAD Therapy ${ }^{\ddagger}$ & $77.6 \%$ & $36.1 \%$ & 0.92 & $66.7 \%$ & $23.2 \%$ & 0.97 \\
\hline Alpha-glucosidase inhibitors & $0.8 \%$ & $0.3 \%$ & 0.08 & $0.4 \%$ & $0.1 \%$ & 0.05 \\
\hline Acarbose & $0.7 \%$ & $0.2 \%$ & 0.07 & $0.4 \%$ & $0.1 \%$ & 0.05 \\
\hline Miglitol & $0.1 \%$ & $0.0 \%$ & 0.03 & $0.0 \%$ & $0.0 \%$ & 0.02 \\
\hline Biguanide: Metformin & $51.4 \%$ & $37.4 \%$ & 0.29 & $51.9 \%$ & $42.6 \%$ & 0.19 \\
\hline Meglitinides & $3.0 \%$ & $1.1 \%$ & 0.13 & $1.4 \%$ & $0.4 \%$ & 0.10 \\
\hline Nateglinide & $1.5 \%$ & $0.5 \%$ & 0.10 & $0.8 \%$ & $0.2 \%$ & 0.08 \\
\hline Repaglinide & $1.6 \%$ & $0.7 \%$ & 0.09 & $0.7 \%$ & $0.2 \%$ & 0.07 \\
\hline Sulfonylureas & $42.5 \%$ & $16.8 \%$ & 0.59 & $28.0 \%$ & $8.7 \%$ & 0.51 \\
\hline Chlorpropamide & $0.0 \%$ & $0.0 \%$ & $<0.01$ & $0.0 \%$ & $0.0 \%$ & 0.01 \\
\hline Glimepiride & $16.5 \%$ & $5.3 \%$ & 0.37 & $12.3 \%$ & $3.2 \%$ & 0.34 \\
\hline Glipizide & $17.7 \%$ & $8.8 \%$ & 0.27 & $11.0 \%$ & $4.3 \%$ & 0.26 \\
\hline Glyburide & $9.5 \%$ & $5.2 \%$ & 0.16 & $5.2 \%$ & $2.0 \%$ & 0.17 \\
\hline Tolazamide & $0.0 \%$ & $0.0 \%$ & 0.01 & $0 \%$ & $0 \%$ & - \\
\hline Tolbutamide & $0.0 \%$ & $0.0 \%$ & $<0.01$ & $0 \%$ & $0 \%$ & - \\
\hline Thiazolidinediones & $24.2 \%$ & $8.4 \%$ & 0.44 & $15.0 \%$ & $5.5 \%$ & 0.32 \\
\hline Pioglitazone & $21.4 \%$ & $7.3 \%$ & 0.41 & $13.6 \%$ & $5.0 \%$ & 0.30 \\
\hline Rosiglitazone & $3.2 \%$ & $1.9 \%$ & 0.08 & $1.5 \%$ & $0.9 \%$ & 0.05 \\
\hline
\end{tabular}

Abbreviations: $\mathrm{OAD}=$ oral anti-diabetic drug; $\mathrm{SD}=$ standard deviation.

${ }^{*}$ Characteristics are presented as percentages unless otherwise indicated.

${ }^{\dagger}$ Matching criteria for which a random sample (without replacement) of up to ten new initiators of non-DPP-4 inhibitor OADs were selected for each saxagliptin initiator.

${ }^{\ddagger}$ Defined as use of an oral anti-diabetic drug within the 180 days prior to the initiation of the index drug. Denominator adjusted to exclude those on index drug.

\section{Discussion}

Drug utilization studies can reveal how medications are administered in clinical practice, identify determinants of drug use, ensure robust prescribing practices [32], and establish topics for further study of drug effectiveness and safety [14]. This study found that across two UK and two US data sources, prior OAD use, hypertension, and hyperlipidemia were associated with initiation of saxagliptin rather than other OADs. Saxagliptin initiation was also associated with hemoglobin A1c results $>8 \%$ within the UK data sources, and a greater number of hemoglobin A1c measurements in the US data sources. Interestingly, saxagliptin was the first OAD utilized for approximately $6 \%$ of patients within the UK data sources, $22 \%$ of patients within US Medicare, and $33 \%$ of patients within the HIRD ${ }^{\text {SM }}$. Results from US Medicare and THIN suggest that saxagliptin may be a preferred treatment in patients with more severe (advanced) T2DM, as evidenced by increased diagnoses for microvascular complications. According to these findings, patients prescribed saxagliptin had higher prevalence of comorbid conditions, poor glycemic control, inadequate response to prior $\mathrm{OAD}$ therapy, or contraindications to OADs in other classes.

Stratifying our analyses on prior OAD use demonstrated that some determinants were more strongly associated with saxagliptin initiation among patients who had not received prior OAD therapy, particularly within 
Table 3 Determinants of saxagliptin use among type 2 diabetes mellitus patients within United Kingdom data sources

\begin{tabular}{|c|c|c|c|c|c|c|}
\hline \multirow{4}{*}{ Characteristic } & \multicolumn{6}{|c|}{ Adjusted odds ratio (95\% confidence interval) $)^{*}$} \\
\hline & \multicolumn{3}{|c|}{ Clinical Practice Research Datalink } & \multicolumn{3}{|c|}{ The Health Improvement Network } \\
\hline & Overall & $\begin{array}{l}\text { Prior OAD } \\
\text { use }\end{array}$ & $\begin{array}{l}\text { No Prior OAD } \\
\text { use }\end{array}$ & Overall & $\begin{array}{l}\text { Prior OAD } \\
\text { use }\end{array}$ & $\begin{array}{l}\text { No Prior OAD } \\
\text { use }\end{array}$ \\
\hline & $(n=21,446)$ & $(n=8,332)$ & $(n=890)$ & $(n=22,020)$ & $(n=8,621)$ & $(n=1,137)$ \\
\hline Hemoglobin A1c $>8 \%$ & $1.26(1.13-1.39)$ & $1.21(1.08-1.35)$ & $1.66(1.05-2.63)$ & $1.17(1.06-1.30)$ & $1.12(1.01-1.25)$ & $1.39(0.95-2.03)$ \\
\hline Overweight vs. $<25 \mathrm{~kg} / \mathrm{m}^{2}$ & $0.91(0.78-1.07)$ & $0.87(0.73-1.03)$ & $1.32(0.67-2.62)$ & $1.38(1.15-1.67)$ & $1.44(1.17-1.76)$ & $0.95(0.48-1.87)$ \\
\hline Obese vs. $<25 \mathrm{~kg} / \mathrm{m}^{2}$ & $1.13(0.99-1.29)$ & $1.12(0.97-1.30)$ & $1.61(0.88-2.94)$ & $1.74(1.45-2.09)$ & $1.83(1.50-2.22)$ & $0.86(0.44-1.68)$ \\
\hline Smoking & $0.93(0.81-1.06)$ & $0.94(0.81-1.08)$ & $0.73(0.43-1.26)$ & $1.03(0.93-1.15)$ & $1.03(0.92-1.16)$ & $0.86(0.57-1.30)$ \\
\hline \multicolumn{7}{|l|}{ Severity of type 2 diabetes mellitus } \\
\hline Cardiovascular & $1.05(0.90-1.23)$ & $1.03(0.87-1.22)$ & $1.49(0.74-3.00)$ & $0.97(0.84-1.13)$ & $1.01(0.86-1.18)$ & $0.74(0.43-1.28)$ \\
\hline Cerebrovascular & $0.79(0.64-0.98)$ & $0.80(0.64-1.01)$ & $0.52(0.20-1.38)$ & $0.87(0.72-1.05)$ & $0.89(0.72-1.09)$ & $0.65(0.31-1.40)$ \\
\hline Nephropathy & $1.08(0.78-1.50)$ & $1.05(0.74-1.50)$ & $1.40(0.41-4.80)$ & $1.75(1.33-2.30)$ & $1.77(1.32-2.39)$ & $1.79(0.61-5.23)$ \\
\hline Peripheral vascular disease & $0.88(0.73-1.07)$ & $0.90(0.74-1.10)$ & $0.54(0.21-1.35)$ & $0.83(0.69-1.00)$ & $0.83(0.68-1.01)$ & $1.32(0.65-2.68)$ \\
\hline Retinopathy & $1.11(0.98-1.25)$ & $1.06(0.93-1.20)$ & $2.25(1.27-3.99)$ & $1.20(1.06-1.35)$ & $1.14(1.00-1.29)$ & $2.63(1.64-4.22)$ \\
\hline \multicolumn{7}{|l|}{ Diagnoses } \\
\hline Allergic rhinitis/hay fever & $0.83(0.69-1.00)$ & $0.88(0.73-1.07)$ & $0.36(0.13-0.97)$ & $0.92(0.77-1.11)$ & $0.99(0.82-1.21)$ & $0.52(0.25-1.07)$ \\
\hline Asthma & $0.99(0.83-1.17)$ & $0.96(0.80-1.15)$ & $1.35(0.63-2.86)$ & $0.99(0.84-1.17)$ & $0.96(0.80-1.15)$ & $1.44(0.79-2.64)$ \\
\hline Chronic obstructive pulmonary disease & $1.23(1.03-1.46)$ & $1.18(0.98-1.42)$ & $2.64(1.34-5.18)$ & $1.21(1.03-1.43)$ & $1.21(1.01-1.44)$ & $1.48(0.79-2.77)$ \\
\hline Collagen vascular disease/autoimmune disorders & $0.96(0.82-1.13)$ & $0.94(0.79-1.11)$ & $1.40(0.64-3.07)$ & $1.01(0.86-1.17)$ & $1.03(0.88-1.22)$ & $1.11(0.59-2.12)$ \\
\hline Dermatologic disorders & $1.08(0.96-1.22)$ & $1.12(0.99-1.27)$ & $0.50(0.28-0.89)$ & $1.10(0.98-1.24)$ & $1.14(1.00-1.29)$ & $0.97(0.62-1.52)$ \\
\hline Hyperlipidemia & $1.19(1.03-1.37)$ & $1.14(0.98-1.33)$ & $2.08(1.14-3.81)$ & $1.21(1.05-1.39)$ & $1.18(1.02-1.37)$ & $1.04(0.59-1.83)$ \\
\hline Hypertension & $0.86(0.75-0.98)$ & $0.83(0.73-0.96)$ & $1.14(0.67-1.93)$ & $0.88(0.78-1.00)$ & $0.86(0.75-0.99)$ & $1.39(0.87-2.22)$ \\
\hline Infectious diseases & $1.16(1.01-1.33)$ & $1.14(0.99-1.32)$ & $1.63(0.97-2.76)$ & $1.10(0.96-1.25)$ & $1.08(0.94-1.24)$ & $1.30(0.79-2.13$ \\
\hline Malignancy & $0.98(0.87-1.11)$ & $0.95(0.84-1.08)$ & $1.78(1.08-2.95)$ & $0.95(0.85-1.07)$ & $0.96(0.84-1.09)$ & $0.83(0.53-1.29)$ \\
\hline Obesity & $1.07(0.93-1.23)$ & $0.99(0.85-1.15)$ & $1.47(0.83-2.60)$ & $1.00(0.87-1.16)$ & $0.96(0.83-1.13)$ & $1.76(1.03-3.02)$ \\
\hline Other diseases & $1.08(0.94-1.25)$ & $1.08(0.93-1.26)$ & $0.77(0.40-1.46)$ & $1.12(0.97-1.28)$ & $1.06(0.92-1.23)$ & $1.31(0.78-2.19$ \\
\hline \multicolumn{7}{|l|}{ Drugs } \\
\hline Acetaminophen/paracetamol & $0.95(0.84-1.07)$ & $0.96(0.84-1.09)$ & $0.80(0.44-1.46)$ & $0.92(0.82-1.03)$ & $0.88(0.78-1.00)$ & $1.18(0.74-1.87)$ \\
\hline Anti-asthmatic agents & $0.96(0.81-1.13)$ & $1.03(0.86-1.23)$ & $0.50(0.23-1.07)$ & $0.92(0.79-1.09)$ & $0.96(0.81-1.14)$ & $0.78(0.43-1.44)$ \\
\hline Antibacterial agents & $1.03(0.92-1.16)$ & $1.00(0.89-1.13)$ & $1.36(0.82-2.26)$ & $0.99(0.88-1.11)$ & $0.98(0.87-1.10)$ & $1.08(0.69-1.69)$ \\
\hline Anticonvulsants & $1.16(0.96-1.40)$ & $1.19(0.98-1.46)$ & $0.87(0.32-2.37)$ & $1.07(0.86-1.33)$ & $1.05(0.83-1.33)$ & $0.95(0.39-2.3$ \\
\hline Antihistamines & $0.93(0.76-1.14)$ & $0.91(0.73-1.12)$ & $1.57(0.61-4.04)$ & $1.01(0.83-1.23)$ & $0.98(0.79-1.22)$ & $1.35(0.65-2.77)$ \\
\hline Antihyperlipidemic agents & $1.33(1.16-1.52)$ & $1.21(1.04-1.39)$ & $2.79(1.60-4.85)$ & $1.32(1.15-1.51)$ & $1.19(1.03-1.38)$ & $2.41(1.50-3.88)$ \\
\hline \multicolumn{7}{|l|}{ Antihypertensive agents } \\
\hline Angiotensin-converting enzyme inhibitors & $1.11(0.98-1.26)$ & $1.12(0.99-1.28)$ & $0.80(0.44-1.47)$ & $1.00(0.88-1.12)$ & $0.98(0.86-1.11)$ & $0.88(0.55-1.41)$ \\
\hline Angiotensin receptor blockers & $1.35(1.16-1.57)$ & $1.36(1.16-1.60)$ & $1.34(0.65-2.75)$ & $1.32(1.14-1.52)$ & $1.29(1.11-1.51)$ & $1.46(0.83-2.58)$ \\
\hline Beta blockers & $1.04(0.90-1.19)$ & $1.03(0.89-1.18)$ & $1.46(0.78-2.72)$ & $1.09(0.96-1.24)$ & $1.05(0.92-1.21)$ & $1.49(0.90-2.45)$ \\
\hline Calcium channel blockers & $1.00(0.88-1.13)$ & $1.04(0.91-1.19)$ & $0.29(0.15-0.58)$ & $0.94(0.84-1.06)$ & $0.99(0.88-1.13)$ & $0.51(0.31-0.86)$ \\
\hline Loop diuretics & $0.95(0.80-1.12)$ & $0.91(0.76-1.09)$ & $1.00(0.46-2.19)$ & $1.40(1.20-1.63)$ & $1.31(1.11-1.55)$ & $1.94(1.07-3.52)$ \\
\hline Other antihypertensive agents & $1.12(0.97-1.29)$ & $0.98(0.81-1.19)$ & $2.03(0.82-5.03)$ & $1.18(1.02-1.36)$ & $0.91(0.74-1.12)$ & $1.73(0.76-3.95)$ \\
\hline Thiazide diuretics & $1.01(0.84-1.22)$ & $1.18(1.02-1.37)$ & $0.39(0.17-0.94)$ & $0.97(0.80-1.18)$ & $1.27(1.10-1.48)$ & $0.54(0.28-1.02)$ \\
\hline Anti-infective agents & $0.97(0.74-1.28)$ & $1.00(0.75-1.34)$ & $0.59(0.13-2.64)$ & $0.78(0.60-1.02)$ & $0.72(0.54-0.95)$ & $0.80(0.29-2.26)$ \\
\hline Antiplatelet/anticoagulant agents & $0.91(0.81-1.03)$ & $0.90(0.79-1.02)$ & $1.16(0.65-2.07)$ & $1.05(0.93-1.18)$ & $1.01(0.89-1.14)$ & $1.53(0.94-2.50)$ \\
\hline
\end{tabular}


Table 3 Determinants of saxagliptin use among type 2 diabetes mellitus patients within United Kingdom data sources (Continued)

\begin{tabular}{llllllll}
\hline Non-steroidal anti-inflammatory agents & $0.98(0.84-1.14)$ & $0.90(0.79-1.02)$ & $0.71(0.30-1.69)$ & $0.98(0.84-1.15)$ & $1.05(0.89-1.24)$ & $0.48(0.24-0.96)$ \\
Other medications & $1.03(0.89-1.19)$ & $1.03(0.89-1.20)$ & $0.63(0.31-1.29)$ & $1.09(0.95-1.25)$ & $1.12(0.97-1.30)$ & $0.79(0.44-1.41)$ \\
Psychotropic agents & $0.94(0.83-1.07)$ & $0.99(0.87-1.13)$ & $0.51(0.25-1.05)$ & $0.95(0.83-1.07)$ & $0.97(0.85-1.11)$ & $0.58(0.34-1.00)$
\end{tabular}

Abbreviations: $\mathrm{OAD}=$ oral anti-diabetic drug.

*Odds ratios adjusted for all other variables in this table.

the UK data sources. One exception was the finding that within THIN, obesity and diabetic nephropathy were more strongly associated with saxagliptin initiation among those with prior OAD use. However, stratifying on prior OAD use reduced the overall sample sizes within each stratum, particularly for patients without prior OAD use. As a result, these findings should be interpreted with caution.

These findings contribute to a growing body of research evaluating the characteristics of patients prescribed DPP-4 inhibitors. In three studies within the Ingenix (now Optum) administrative claims database [33-35], patients treated with sitagliptin, another DPP-4 inhibitor, were more likely to have medical comorbidities (i.e., cardiovascular disease, chronic kidney disease, hypertension, lipid disorders, and neuropathy) and were more frequently prescribed cardiovascular medications and insulin. In two additional studies within the General Electric Healthcare's Clinical Data Services electronic medical records database, patients prescribed sitagliptin were older and had a higher prevalence of preexisting comorbid conditions than patients prescribed other OAD therapies [36]. Patients prescribed sitagliptin were also more likely to have baseline microvascular and macrovascular complications of T2DM than patients receiving exenatide [37]. Our results expand understanding of the DPP-4 drug class by providing new data on determinants associated with saxagliptin initiation and including large samples of T2DM patients within the US and UK.

The observation that saxagliptin was prescribed to a large proportion of T2DM patients without prior OAD use in the US data sources (22\% within US Medicare; $33 \%$ within $\mathrm{HIRD}^{\mathrm{SM}}$ ) compared to the UK data sources (6\%) is surprising given current guidelines recommending use of metformin as first-line OAD therapy [38]. A recent study utilizing the IMS Health Vector One National and Total Patient Tracker databases, a compilation of large commercial outpatient prescription and patient databases in the US, similarly found that $28 \%$ of non-insulin OAD users were not prescribed metformin and that DPP-4 inhibitors were the most commonly prescribed new drug class of agents [39]. The reasons why saxagliptin was more commonly prescribed as initial OAD treatment among T2DM patients within the US data sources remain unclear. Decreased metformin use may be due, in part, to contraindications to the medication (e.g. renal insufficiency, active liver disease) [40-45]. Further studies are needed to evaluate the reasons for this deviation from recommended prescribing practices.

Since baseline characteristics of patients with T2DM have been shown to influence the efficacy of antidiabetic therapy [46,47], it will be important to evaluate the determinants of saxagliptin use identified in this study as effect modifiers and confounders in future comparative effectiveness and safety studies. Our results also provide valuable information on variables that should be considered for inclusion within propensity score analyses of saxagliptin use for future pharmacoepidemiologic studies evaluating the comparative effectiveness and safety of saxagliptin compared to other OADs [18].

A particular strength of our analysis was the inclusion of data from US Medicare. Prior studies that evaluated the characteristics of OAD initiators within the US [33-35,37], but did not include Medicare coverage, likely underrepresented T2DM patients over the age of 65 and may have incompletely captured claims among patients also co-enrolled in Medicare. By examining initiators of saxagliptin and other non-DPP-4 inhibitor OADs within four data sources (including US Medicare) and across two continents, our analyses ensured adequate capture and representation of elderly T2DM patients.

Our study has several potential limitations. First, we were unable to determine the duration of T2DM due to the use of administrative data (US data sources) and incomplete electronic health data from patients who may have switched practices (UK data sources). Second, actual exposure to saxagliptin and other OADs cannot be confirmed. However, minimal misclassification of medication use is expected since prescribing records within the UK data sources and pharmacy claims within the US data sources were used to determine drug exposure. Additionally, all relevant diagnosis and procedure codes were included and reviewed by clinical and pharmacoepidemiology experts to minimize misclassification of medical comorbidities examined as determinants of saxagliptin use. Third, some potentially important variables, including alcohol and illicit drug use, 
Table 4 Determinants of saxagliptin use among type 2 diabetes mellitus patients within United States data sources

\begin{tabular}{|c|c|c|c|c|c|c|}
\hline \multirow{3}{*}{ Characteristic } & \multicolumn{6}{|c|}{ Adjusted odds ratio ( $95 \%$ confidence interval) ${ }^{*}$} \\
\hline & \multicolumn{3}{|l|}{ US Medicare } & \multicolumn{3}{|c|}{ HealthCore Integrated Research Database $\mathrm{SM}^{\mathrm{SM}}$} \\
\hline & Overall & $\begin{array}{l}\text { Prior OAD } \\
\text { use }\end{array}$ & $\begin{array}{l}\text { No prior OAD } \\
\text { use }\end{array}$ & Overall & $\begin{array}{l}\text { Prior OAD } \\
\text { use }\end{array}$ & $\begin{array}{l}\text { No prior } \\
\text { OAD use }\end{array}$ \\
\hline & $(n=545,408)$ & $(n=177,791)$ & $(n=82,840)$ & $(n=85,865)$ & $(n=17,177)$ & $(n=22,803)$ \\
\hline
\end{tabular}

Number of hemoglobin A1c measurements

1 vs. 0 measured

$2+$ vs. 0 measured

Severity of type 2 diabetes mellitus

Cardiovascular

Cerebrovascular

Metabolic (ketoacidosis, hyperosmolar, coma)

Nephropathy

Neuropathy

Peripheral vascular disease

Retinopathy

Unspecified additional diabetic complications $^{\dagger}$

\section{Diagnoses}

Allergic rhinitis/hay fever

Asthma

Chronic obstructive pulmonary disease

Collagen vascular disease/ autoimmune disorders Rheumatoid arthritis ${ }^{\ddagger}$

Spondyloarthritis ${ }^{\ddagger}$

Dermatologic disorders

Psoriasis $^{\ddagger}$

Hyperlipidemia

Hypertension

Infectious diseases

Cellulitis $^{\ddagger}$

Malignancy

Obesity

Other diseases

Alcohol diseases ${ }^{\ddagger}$

Gastrointestinal diseases ${ }^{\ddagger}$

Neurological diseases ${ }^{\ddagger}$

\section{Drugs}

Acetaminophen/paracetamol

Anti-asthmatic agents

Antibacterial agents

Anticonvulsants

Antihistamines

Antihyperlipidemic agents

Antihypertensive agents

Angiotensin-converting enzyme inhibitors Angiotensin receptor blockers
$2.10(2.04-2.16) \quad 2.09(2.02-2.16) \quad 2.02(1.91-2.12) \quad 1.67(1.56-1.78) \quad 1.40(1.27-1.54) \quad 1.96(1.77-2.17)$

$2.93(2.85-3.01) \quad 2.87(2.77-2.97) \quad 2.95(2.78-3.12) \quad 2.33(2.16-2.51) \quad 1.91(1.72-2.12) \quad 1.96(1.77-2.17)$

$0.95(0.93-0.97) \quad 0.95(0.93-0.98) \quad 0.95(0.90-1.00) \quad 0.96(0.88-1.05) \quad 0.97(0.86-1.10) \quad 1.00(0.76-1.32)$ $0.90(0.88-0.93) \quad 0.92(0.88-0.95) \quad 0.87(0.82-0.94) \quad 0.92(0.78-1.09) \quad 0.93(0.74-1.17) \quad 0.90(0.77-1.06)$ $0.95(0.87-1.03) \quad 0.91(0.82-1.01) \quad 1.06(0.89-1.27)-$

$1.12(1.09-1.15) \quad 1.08(1.05-1.12) \quad 1.25(1.18-1.32) \quad 1.04(0.93-1.18) \quad 0.87(0.75-1.02) \quad 1.40(1.14-1.72)$ $1.11(1.08-1.14) \quad 1.08(1.05-1.11) \quad 1.22(1.15-1.28) \quad 1.01(0.92-1.11) \quad 1.05(0.92-1.19) \quad 1.03(0.87-1.22)$ $0.97(0.94-1.00) \quad 0.97(0.94-1.01) \quad 0.96(0.91-1.02) \quad 1.06(0.92-1.21) \quad 0.92(0.77-1.11) \quad 1.30(1.03-1.63)$ $1.03(1.00-1.06) \quad 1.01(0.97-1.04) \quad 1.09(1.02-1.16) \quad 1.02(0.91-1.14) \quad 0.95(0.82-1.10) \quad 1.11(0.90-1.37)$ $0.96(0.92-0.99) \quad 0.91(0.82-1.01) \quad 1.12(1.04-1.22) \quad 1.01(0.89-1.14) \quad 0.98(0.83-1.16) \quad 1.17(0.93-1.47)$ $1.16(1.12-1.21) \quad 1.19(1.13-1.25) \quad 1.14(1.05-1.23) \quad 1.12(1.00-1.26) \quad 1.29(1.09-1.52) \quad 0.95(0.78-1.15)$ $0.91(0.87-0.94) \quad 0.89(0.85-0.94) \quad 0.94(0.87-1.02) \quad 0.83(0.72-0.95) \quad 0.92(0.76-1.13) \quad 0.80(0.63-1.02)$ $0.92(0.89-0.95) \quad 0.92(0.88-0.95) \quad 0.89(0.83-0.95) \quad 0.93(0.79-1.08) \quad 0.88(0.70-1.09) \quad 0.93(0.72-1.21)$ $1.01(0.95-1.08) \quad 1.05(0.97-1.14) \quad 0.93(0.82-1.06) \quad 0.90(0.79-1.03) \quad 0.94(0.79-1.12) \quad 0.82(0.66-1.03)$ $1.10(1.03-1.16) \quad 1.08(1.00-1.17) \quad 1.17(1.04-1.32) \quad-$ $1.04(1.00-1.09) \quad 1.05(1.00-1.10) \quad 1.03(0.95-1.12) \quad-$

$1.04(0.99-1.10) \quad 1.02(0.96-1.09) \quad 1.08(0.98-1.20) \quad 1.07(0.93-1.23) \quad 1.10(0.90-1.34) \quad 1.14(0.90-1.44)$ $0.97(0.88-1.08) \quad 0.99(0.87-1.12) \quad 0.86(0.70-1.06) \quad-$

1.11 (1.08-1.14) $1.11(1.08-1.15) \quad 1.11(1.05-1.17) \quad 1.17(1.10-1.24) \quad 1.17(1.08-1.27) \quad 1.11$ (1.01-1.22) $1.00(0.97-1.03) \quad 1.01(0.97-1.04) \quad 0.99(0.93-1.05) \quad 1.13(1.07-1.20) \quad 1.17(1.08-1.27) \quad 1.18(1.07-1.31)$ $0.86(0.79-0.94) \quad 0.88(0.79-0.99) \quad 0.85(0.71-1.02) \quad 0.89(0.79-1.01) \quad 1.17(1.08-1.27) \quad 0.95(0.78-1.16)$ $0.92(0.88-0.95) \quad 0.91(0.87-0.95) \quad 0.92(0.85-1.00) \quad-$

$0.93(0.90-0.97) \quad 0.95(0.91-0.99) \quad 0.89(0.83-0.96) \quad 1.02(0.90-1.17) \quad 1.17(1.08-1.27) \quad 1.03(0.83-1.27)$ $0.94(0.91-0.97) \quad 0.96(0.92-0.99) \quad 0.90(0.84-0.97) \quad 0.88(0.81-0.96) \quad 0.95(0.84-1.07) \quad 0.76(0.65-0.88)$

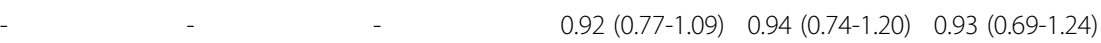
$0.68(0.61-0.76) \quad 0.71(0.62-0.81) \quad 0.90(0.84-0.97) \quad-$ $1.01(0.95-1.06) \quad 0.98(0.91-1.05) \quad 1.07(0.95-1.20) \quad-$ $0.76(0.63-0.93) \quad 0.86(0.68-1.08) \quad 0.53(0.35-0.82) \quad-$

$0.97(0.94-0.99) \quad 0.97(0.94-1.00) \quad 0.96(0.91-1.01) \quad 0.97(0.91-1.04) \quad 1.02(0.93-1.12) \quad 0.87(0.78-0.98)$ $1.08(1.05-1.12) \quad 1.09(1.05-1.14) \quad 1.08(1.01-1.16) \quad 1.02(0.93-1.13) \quad 1.02(0.88-1.17) \quad 1.06(0.89-1.25)$ $1.08(1.06-1.10) \quad 1.08(1.05-1.11) \quad 1.08(1.03-1.13) \quad 1.07(1.01-1.13) \quad 1.12(1.04-1.21) \quad 0.95$ (0.87-1.04) $0.89(0.85-0.93) \quad 0.91(0.87-0.96) \quad 0.81(0.74-0.90) \quad 0.96(0.87-1.06) \quad 1.01(0.88-1.16) \quad 0.89(0.75-1.07)$ $1.10(1.07-1.14) \quad 1.10(1.06-1.15) \quad 1.09(1.01-1.17) \quad 1.05(0.94-1.16) \quad 1.05(0.91-1.22) \quad 1.11$ (0.93-1.32) $1.22(1.19-1.24) \quad 1.15(1.12-1.19) \quad 1.35(1.29-1.41) \quad 1.13(1.07-1.19) \quad 1.11(1.03-1.20) \quad 1.16(1.06-1.28)$

$0.98(0.96-1.01) \quad 0.96(0.94-0.99) \quad 1.00(0.96-1.05) \quad 0.97(0.91-1.02) \quad 0.92(0.85-1.00) \quad 1.01(0.90-1.12)$ $1.39(1.36-1.43) \quad 1.32(1.28-1.36) \quad 1.59(1.51-1.68) \quad 1.22(1.13-1.31) \quad 1.07(0.97-1.19) \quad 1.44(1.26-1.65)$ 
Table 4 Determinants of saxagliptin use among type 2 diabetes mellitus patients within United States data sources (Continued)

\begin{tabular}{|c|c|c|c|c|c|c|}
\hline Beta blockers & $1.04(1.02-1.06)$ & $1.05(1.02-1.08)$ & $1.02(0.98-1.07)$ & $0.99(0.93-1.06)$ & $1.10(1.00-1.20)$ & $0.86(0.76-0.97)$ \\
\hline Calcium channel blockers & $1.02(0.99-1.04)$ & $1.01(0.98-1.04)$ & $1.02(0.97-1.07)$ & $0.97(0.90-1.04)$ & $1.04(0.94-1.14)$ & $0.86(0.76-0.99)$ \\
\hline Loop diuretics & $1.10(1.07-1.13)$ & $1.09(1.05-1.12)$ & $1.13(1.07-1.20)$ & $0.94(0.83-1.06)$ & $0.92(0.79-1.08)$ & $0.99(0.80-1.22)$ \\
\hline Other antihypertensive agents & $1.11(1.07-1.14)$ & $1.11(1.07-1.15)$ & $1.09(1.02-1.17)$ & $1.06(0.94-1.20)$ & $1.05(0.96-1.16)$ & $0.99(0.79-1.24)$ \\
\hline Thiazidediuretics & $1.14(1.11-1.17)$ & $1.14(1.11-1.18)$ & $1.14(1.08-1.21)$ & $0.99(0.93-1.07)$ & $1.05(0.96-1.16)$ & $0.83(0.73-0.95)$ \\
\hline Anti-infective agents & - & - & - & $1.04(0.95-1.14)$ & $1.00(0.88-1.14)$ & $1.01(0.86-1.18)$ \\
\hline Antifungals $^{\ddagger}$ & $1.10(1.06-1.14)$ & $1.06(1.02-1.10)$ & $1.21(1.13-1.30)$ & - & - & - \\
\hline Antivirals $^{\ddagger}$ & $1.09(1.02-1.16)$ & $1.10(1.02-1.19)$ & $1.07(0.93-1.23)$ & - & - & - \\
\hline Antiplatelet/anticoagulant agents & $0.94(0.86-1.02)$ & $0.97(0.87-1.07)$ & $0.91(0.75-1.10)$ & $0.98(0.88-1.10)$ & $0.98(0.84-1.13)$ & $1.07(0.87-1.32)$ \\
\hline Clopidogrel $^{\dagger}$ & $1.13(1.10-1.17)$ & $1.12(1.08-1.16)$ & $1.18(1.11-1.26)$ & - & - & - \\
\hline Warfarin $^{+}$ & $0.96(0.92-0.99)$ & $0.99(0.94-1.04)$ & $0.85(0.78-0.93)$ & - & - & - \\
\hline Non-steroidal anti-inflammatory agents & $1.06(1.03-1.09)$ & $1.05(1.02-1.09)$ & $1.09(1.03-1.15)$ & $1.02(0.95-1.10)$ & $1.05(0.94-1.16)$ & $0.99(0.87-1.12)$ \\
\hline Other medications & $1.06(1.01-1.12)$ & $1.08(1.01-1.15)$ & $1.08(0.96-1.22)$ & $0.88(0.81-0.96)$ & $0.83(0.75-0.93)$ & $0.99(0.85-1.14)$ \\
\hline Allopurinol $^{\ddagger}$ & $1.05(1.01-1.10)$ & $1.06(1.00-1.11)$ & $1.07(0.97-1.18)$ & - & - & - \\
\hline Anti-arrhythmics $^{\ddagger}$ & $0.99(0.96-1.02)$ & $0.99(0.95-1.02)$ & $1.00(0.94-1.08)$ & - & - & - \\
\hline Immune modulators/ suppressants $^{\ddagger}$ & $1.05(1.00-1.10)$ & $1.01(0.95-1.07)$ & $1.16(1.04-1.28)$ & - & - & - \\
\hline Nitroglycerin $^{\ddagger}$ & $0.98(0.94-1.03)$ & $0.97(0.92-1.03)$ & $1.01(0.92-1.13)$ & - & - & - \\
\hline Urinary anti-spasmodics $^{\ddagger}$ & $1.03(0.98-1.07)$ & $1.03(0.98-1.09)$ & $1.05(0.96-1.16)$ & - & - & - \\
\hline Psychotropic agents & $0.93(0.91-0.95)$ & $0.92(0.89-0.94)$ & $0.92(0.87-0.96)$ & $0.94(0.88-1.00)$ & $0.95(0.87-1.04)$ & $0.87(0.77-0.97)$ \\
\hline
\end{tabular}

Abbreviations: $\mathrm{OAD}=$ oral anti-diabetic drug.

*Odds ratios adjusted for all other variables in this table.

${ }^{\dagger}$ For HIRD ${ }^{S M}$, due to low prevalence, metabolic complications are included in the analyses of unspecified additional diabetic complications.

${ }^{\ddagger}$ Medications and diseases were evaluated separately within US Medicare because of the large sample size within this data source.

diet, exercise, family history of diseases, and nonprescription drug use, were not recorded within the data sources. Finally, our results may not be generalizable to all settings. However, our analyses have expanded the populations to which these findings can be generalized by examining results from four different data sources within the US and UK [48], which contain claims and medical records data from both private and public health insurance plans.

\section{Conclusion}

In summary, this study found that saxagliptin initiation was more common in patients with prior complications associated with T2DM, prior OAD use, and diagnoses and receipt of treatment for hyperlipidemia and hypertension. These variables should be considered in future studies evaluating the comparative safety and effectiveness of saxagliptin and other OADs.

\section{Abbreviations}

CPRD: Clinical Practice Research Datalink; DPP-4: Dipeptidyl peptidase-4; FDA: US Food and Drug Administration; HIRD ${ }^{S M}$ : HealthCore Integrated Research Database ${ }^{\text {SM; }}$ OAD: Oral anti-diabetic drug; T2DM: Type 2 diabetes mellitus; THIN: The Health Improvement Network; UK: United Kingdom; US: United States.

\section{Competing interests}

These series of studies are funded by AstraZeneca and Bristol-Myers Squibb. The study's sponsors approved the protocol and have the right to provide non-binding comments on resulting reports and manuscripts. All authors receive funding from AstraZeneca and Bristol-Myers Squibb through their employers. Further, Dr. Lo Re has received grant support (through the University of Pennsylvania) from Gilead Sciences, unrelated to oral anti-diabetic drugs. Dr. Hennessy has done consulting for AstraZeneca, unrelated to saxagliptin. Dr. Haynes, Dr. Holick, and Ms. Esposito are employed by HealthCore, Inc., and Ms. Bhullar is employed by CSD Medical Research. Each of these individuals' respective organizations conducts research for and receives funding from pharmaceutical manufacturers for its research services. Dr. Strom has done consulting for AstraZeneca and Bristol-Myers Squibb.

\section{Authors' contributions}

MES, DMC, CWN, JAR, MSN, KH, BLS, and VLR participated in the conception and design of the study. $C N H, D B E, A G$, and $H B$ participated in data collection. CWN and JAR performed data management and analysis. MES, DMC, CWN, MSN, KH, and VLR collaborated on the initial drafts of the manuscript. All other authors contributed further to interpretation of the results and manuscript edits. All authors read and approved the final manuscript.

\section{Acknowledgements}

The authors would like to thank Jennifer Wood, PhD, MPH of Bristol-Myers Squibb and Laura Horne, MHS of AstraZeneca for their assistance with the HeathCore Integrated Research Database ${ }^{\mathrm{SM}}$, Clinical Practice Research Datalink, and The Health Improvement Network data sources. Industry sponsors were excluded from all analyses involving US Medicare data. The authors would also like to thank Cristin P. Freeman, MPH, MBE for her assistance in the ascertainment and use of US Medicare Data. 


\section{Author details}

'Department of Biostatistics and Epidemiology, Center for Clinical Epidemiology and Biostatistics, Perelman School of Medicine at the University of Pennsylvania, 423 Guardian Drive, Philadelphia, PA, USA. ${ }^{2}$ Department of Biostatistics and Epidemiology, Center for Pharmacoepidemiology Research and Training, Perelman School of Medicine at the University of Pennsylvania, Philadelphia, PA, USA. ${ }^{3}$ HealthCore, Inc, Wilmington, DE, USA. ${ }^{4}$ Department of Medicine, Perelman School of Medicine at the University of Pennsylvania, Philadelphia, PA, USA. ${ }^{5} \mathrm{Clinical}$ Practice Research Datalink, Medicines and Healthcare Products Regulatory Agency, London, UK. ${ }^{6}$ Cegedim Strategic Data Medical Research, London, UK. ${ }^{7}$ Rutgers Biomedical \& Health Sciences, Rutgers, the State University of New Jersey, Newark, NJ, USA.

Received: 15 December 2014 Accepted: 16 March 2015 Published online: 02 April 2015

\section{References}

1. Danaei G, Finucane MM, Lu Y, Singh GM, Cowan MJ, Paciorek CJ, et al. National, regional, and global trends in fasting plasma glucose and diabetes prevalence since 1980: systematic analysis of health examination surveys and epidemiological studies with 370 country-years and 2.7 million participants. Lancet. 2011;378:31-40.

2. Wild S, Roglic G, Green A, Sicree R, King H. Global prevalence of diabetes: estimates for the year 2000 and projections for 2030. Diabetes Care. 2004:27:1047-53.

3. Gonzalez EL, Johansson S, Wallander MA, Rodriguez LA. Trends in the prevalence and incidence of diabetes in the UK: 1996-2005. J Epidemiol Community Health. 2009;63:332-6.

4. Centers for Disease Control and Prevention. National Diabetes Statistics Report: Estimates of Diabetes and Its Burden in the United States, 2014. Atlanta, GA: U.S. Department for Health and Human Services; 2014.

5. Huang ES, Basu A, O'Grady M, Capretta JC. Projecting the future diabetes population size and related costs for the U.S. Diabetes Care. 2009;32:2225-9.

6. Bolen S, Feldman L, Vassy J, Wilson L, Yeh HC, Marinopoulos S, et al. Systematic review: comparative effectiveness and safety of oral medications for type 2 diabetes mellitus. Ann Intern Med. 2007;147:386-99.

7. Tahrani AA, Piya MK, Barnett AH. Saxagliptin: a new DPP-4 inhibitor for the treatment of type 2 diabetes mellitus. Adv Ther. 2009;26:249-62.

8. Rosenstock J, Sankoh S, List JF. Glucose-lowering activity of the dipeptidyl peptidase-4 inhibitor saxagliptin in drug-naive patients with type 2 diabetes. Diabetes Obes Metab. 2008;10:376-86.

9. Jadzinsky M, Pfutzner A, Paz-Pacheco E, Xu Z, Allen E, Chen R. Saxagliptin given in combination with metformin as initial therapy improves glycaemic control in patients with type 2 diabetes compared with either monotherapy: a randomized controlled trial. Diabetes Obes Metab. 2009;11:611-22.

10. DeFronzo RA, Hissa MN, Garber AJ, Luiz Gross J, Yuyan Duan R, Ravichandran S, et al. The efficacy and safety of saxagliptin when added to metformin therapy in patients with inadequately controlled type 2 diabetes with metformin alone. Diabetes Care. 2009;32:1649-55.

11. Chacra AR, Tan GH, Apanovitch A, Ravichandran S, List J, Chen R. Saxagliptin added to a submaximal dose of sulphonylurea improves glycaemic control compared with uptitration of sulphonylurea in patients with type 2 diabetes: a randomised controlled trial. Int J Clin Pract. 2009;63:1395-406.

12. Hollander P, Li J, Allen E, Chen R, Investigators C. Saxagliptin added to a thiazolidinedione improves glycemic control in patients with type 2 diabetes and inadequate control on thiazolidinedione alone. J Clin Endocrinol Metab. 2009;94:4810-9.

13. Barnett AH, Charbonnel B, Donovan M, Fleming D, Chen R. Effect of saxagliptin as add-on therapy in patients with poorly controlled type 2 diabetes on insulin alone or insulin combined with metformin. Curr Med Res Opin. 2012;28:513-23.

14. SC Pradhan DGS, CH Shashindran, JS Bapna. Drug utilization studies. Natl Med J India 1988, 1:185-189.

15. Khan GH, Aqil M, Pillai KK, Ahmad MA, Kapur P, Ain MR, et al. Therapeutic adherence: a prospective drug utilization study of oral hypoglycemic in patients with type 2 diabetes mellitus. Asian Pac J Trop Dis. 2014;4(Supplement 1):S347-52.

16. Walker AM. Confounding by indication. Epidemiology. 1996;7:335-6.
17. Glynn RJ, Schneeweiss S, Stürmer T. Indications for propensity scores and review of their use in pharmacoepidemiology. Basic Clin Pharmacol Toxicol. 2006;98:253-9.

18. Lo Re V 3rd, Haynes K, Ming EE, Wood Ives J, Horne LN, Fortier K, et al. Safety of saxagliptin: rationale for and design of a series of postmarketing observational studies. Pharmacoepidemiol Drug Saf. 2012;21(11):202-15.

19. Williams T, van Staa T, Puri S, Eaton S. Recent advances in the utility and use of the General Practice Research Database as an example of a UK Primary Care Data resource. Ther Adv Drug Saf. 2012;3:89-99.

20. Horsfall $\amalg$, Nazareth I, Petersen I. Serum uric acid and the risk of respiratory disease: a population-based cohort study. Thorax. 2014;69:1021-6.

21. Fett N, Haynes K, Propert KJ, Margolis DJ. Five-year malignancy incidence in patients with chronic pruritus: a population-based cohort study aimed at limiting unnecessary screening practices. J Am Acad Dermatol. 2014;70:651-8

22. Lewis JD, Schinnar R, Bilker WB, Wang X, Strom BL. Validation studies of the health improvement network (THIN) database for pharmacoepidemiology research. Pharmacoepidemiol Drug Saf. 2007;16:393-401.

23. Lo Re V 3rd, Haynes K, Forde KA, Localio AR, Schinnar R, Lewis JD. Validity of The Health Improvement Network (THIN) for epidemiologic studies of hepatitis C virus infection. Pharmacoepidemiol Drug Saf. 2009:18:807-14.

24. Cai B, Xu W, Bortnichak E, Watson DJ. An algorithm to identify medical practices common to both the General Practice Research Database and The Health Improvement Network database. Pharmacoepidemiol Drug Saf. 2012;21:770-4

25. Chan KA, Truman A, Gurwitz JH, Hurley JS, Martinson B, Platt R, et al. A cohort study of the incidence of serious acute liver injury in diabetic patients treated with hypoglycemic agents. Arch Intern Med. 2003;163:728-34.

26. Bullano MF, McNeeley BJ, Yu YF, Quimbo R, Burawski LP, Yu EB, et al. Comparison of costs associated with the use of etanercept, infliximab, and adalimumab for the treatment of rheumatoid arthritis. Manag Care Interface. 2006;19:47-53.

27. Sarawate C, Sikirica MV, Willey VJ, Bullano MF, Hauch O. Monitoring anticoagulation in atrial fibrillation. J Thromb Thrombolysis. 2006;21:191-8.

28. Kamat SA, Gandhi SK, Davidson M. Comparative effectiveness of rosuvastatin versus other statin therapies in patients at increased risk of failure to achieve low-density lipoprotein goals. Curr Med Res Opin. 2007;23:1121-30

29. Young BA, Lin E, Von Korff M, Simon G, Ciechanowski P, Ludman EJ, et al. Diabetes complications severity index and risk of mortality, hospitalization, and healthcare utilization. Am J Manag Care. 2008;14:15-23.

30. American Diabetes Association. Standards of medical care in diabetes-2014. Diabetes Care. 2014;37(Supplement 1):S14-S280.

31. Mamdani M, Sykora K, Li P, Normand S-LT, Streiner DL, Austin PC, et al. Reader's guide to critical appraisal of cohort studies: 2. Assessing potential for confounding. BMJ: British Medical Journal. 2005:330:960-2.

32. Lee D, Bergman U. Studies of Drug Utilization. Pharmacoepidemiology. Fifth edn. Oxford, UK: John Wiley \& Sons, Ltd.; 2012.

33. Dore DD, Seeger JD, Arnold Chan K. Use of a claims-based active drug safety surveillance system to assess the risk of acute pancreatitis with exenatide or sitagliptin compared to metformin or glyburide. Curr Med Res Opin. 2009;25:1019-27.

34. Cai B, Katz L, Alexander CM, Williams-Herman D, Girman CJ. Characteristics of patients prescribed sitagliptin and other oral antihyperglycaemic agents in a large US claims database. Int J Clin Pract. 2010;64:1601-8.

35. Brodovicz KG, Kou TD, Alexander CM, O'Neill EA, Senderak M, Engel SS, et al. Recent trends in the characteristics of patients prescribed sitagliptin and other oral antihyperglycaemic agents in a large U.S. claims database. Int J Clin Pract. 2013:67:449-54.

36. Zhang Q, Rajagopalan S, Mavros P, Engel SS, Davies MJ, Yin D, et al. Baseline characteristic differences between patients prescribed sitagliptin vs. other oral antihyperglycemic agents: analysis of a US electronic medical record database. Curr Med Res Opin. 2010;26:1697-703.

37. Zhang Q, Rajagopalan S, Mavros P, Engel SS, Davies MJ, Yin D, et al. Differences in baseline characteristics between patients prescribed sitagliptin versus exenatide based on a US electronic medical record database. Adv Ther. 2010;27:223-32.

38. Inzucchi SE, Bergenstal RM, Buse JB, Diamant M, Ferrannini E, Nauck M, et al. Management of hyperglycemia in type 2 diabetes: a patient-centered approach: position statement of the American Diabetes Association (ADA) and the 
European Association for the Study of Diabetes (EASD). Diabetes Care. 2012;35:1364-79.

39. Hampp C, Borders-Hemphill V, Moeny DG, Wysowski DK. Use of antidiabetic drugs in the U.S., 2003-2012. Diabetes Care. 2014;37:1367-74.

40. Flory JH, Hennessy S. Metformin Use Reduction in Mild to Moderate Renal Impairment: Possible Inappropriate Curbing of Use Based on Food and Drug Administration Contraindicaitons. JAMA Intern Med. 2014.

41. Sulkin TV, Bosman D, Krentz AJ. Contraindications to metformin therapy in patients with NIDDM. Diabetes Care. 1997;20:925-8.

42. Calabrese AT, Coley KC, DaPos SV, Swanson D, Rao RH. Evaluation of prescribing practices: risk of lactic acidosis with metformin therapy. Arch Intern Med. 2002;162:434-7.

43. Kennedy L, Herman WH. Renal status among patients using metformin in a primary care setting. Diabetes Care. 2005;28:922-4.

44. Vasisht KP, Chen SC, Peng Y, Bakris GL. Limitations of metformin use in patients with kidney disease: are they warranted? Diabetes Obes Metab. 2010;12:1079-83.

45. Horlen C, Malone R, Bryant B, Dennis B, Carey T, Pignone M, et al. Frequency of inappropriate metformin prescriptions. JAMA. 2002;287:2504-5.

46. Bloomgarden ZT, Dodis R, Viscoli CM, Holmboe ES, Inzucchi SE. Lower baseline glycemia reduces apparent oral agent glucose-lowering efficacy: a meta-regression analysis. Diabetes Care. 2006;29:2137-9.

47. Chapell R, Gould AL, Alexander CM. Baseline differences in A1C explain apparent differences in efficacy of sitagliptin, rosiglitazone and pioglitazone. Diabetes Obes Metab. 2009;11:1009-16.

48. Hall GC, Sauer B, Bourke A, Brown JS, Reynolds MW, Casale RL. Guidelines for good database selection and use in pharmacoepidemiology research. Pharmacoepidemiol Drug Saf. 2012;21:1-10.

\section{Submit your next manuscript to BioMed Central and take full advantage of:}

- Convenient online submission

- Thorough peer review

- No space constraints or color figure charges

- Immediate publication on acceptance

- Inclusion in PubMed, CAS, Scopus and Google Scholar

- Research which is freely available for redistribution 